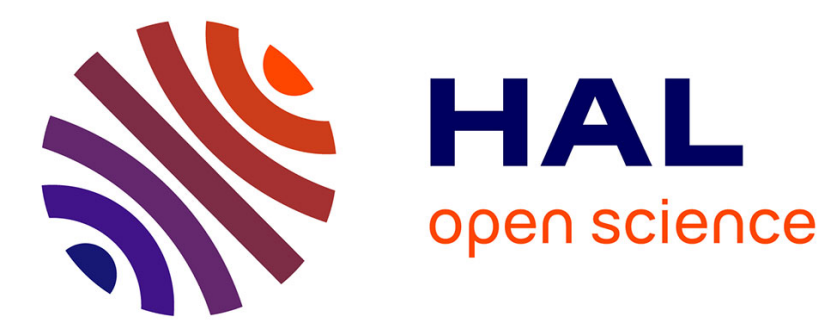

\title{
Mean first-passage times in confined media: from Markovian to non-Markovian processes
}

\author{
Olivier Benichou, Thomas Guérin, Raphaël Voituriez
}

\section{To cite this version:}

Olivier Benichou, Thomas Guérin, Raphä̈l Voituriez. Mean first-passage times in confined media: from Markovian to non-Markovian processes. Journal of Physics A: Mathematical and Theoretical, 2015, 48 (16), pp.1630001 (1-43). 10.1088/1751-8113/48/16/163001 . hal-01146165

\section{HAL Id: hal-01146165 https://hal.science/hal-01146165}

Submitted on 27 Apr 2015

HAL is a multi-disciplinary open access archive for the deposit and dissemination of scientific research documents, whether they are published or not. The documents may come from teaching and research institutions in France or abroad, or from public or private research centers.
L'archive ouverte pluridisciplinaire HAL, est destinée au dépôt et à la diffusion de documents scientifiques de niveau recherche, publiés ou non, émanant des établissements d'enseignement et de recherche français ou étrangers, des laboratoires publics ou privés. 


\title{
Mean first-passage times in confined media: from Markovian to non-Markovian processes
}

\author{
O Bénichou ${ }^{1}$, T Guérin $^{2}$ and R Voituriez ${ }^{3}$ \\ ${ }^{1}$ Laboratoire de Physique Théorique de la Matière Condensée, CNRS UMR 7600, case \\ courrier 121, Université Paris 6, 4 Place Jussieu, 75255 Paris Cedex France \\ ${ }^{2}$ Laboratoire Ondes et Matière d'Aquitaine, University of Bordeaux, Unité Mixte de \\ Recherche 5798, CNRS, F 33400 Talence, France \\ ${ }^{3}$ Laboratoire de Physique Théorique de la Matière Condensée, CNRS UMR 7600, case \\ courrier 121, Université Paris 6, 4 Place Jussieu, 75255 Paris Cedex France
}

\begin{abstract}
We review recent theoretical works that enable the accurate evaluation of the mean first passage time (MFPT) of a random walker to a target in confinement for Markovian (memory-less) and non-Markovian walkers. For the Markovian problem, we present a general theory which allows one to accurately evaluate the MFPT and its extensions to related first-passage observables such as splitting probabilities and occupation times. We show that this analytical approach provides a universal scaling dependence of the MFPT on both the volume of the confining domain and the source target distance in the case of general scale-invariant processes. This analysis is applicable to a broad range of stochastic processes characterized by length scale-invariant properties, and reveals the key role that can be played by the starting position of the random walker. We then present an extension to non-Markovian walks by taking the specific example of a tagged monomer of a polymer chain looking for a target in confinement. We show that the MFPT can be calculated accurately by computing the distribution of the positions of all the monomers in the chain at the instant of reaction. Such a theory can be used to derive asymptotic relations that generalize the scaling dependence with the volume and the initial distance to the target derived for Markovian walks. Finally, we present an application of this theory to the problem of the first contact time between the two ends of a polymer chain, and review the various theoretical approaches of this nonMarkovian problem.
\end{abstract}

Keywords: random walks, first-passage times, stochastic processes 


\section{Introduction}

How long does it take for a drunkard to go from a given bar to another one? This time is known in the random walk literature as a first passage time (FPT) and it has generated a considerable amount of work for many years [1 3 ]. The importance of FPT relies on the fact that many physical properties, including fluorescence quenching [4], neuron dynamics [5] or resonant activation [6] to name a few, are controlled by first passage events. More recently, FPTs have regained interest in the context of random search strategies [7 11].

Generically, one might consider the problem of a random walker whose position $\mathbf{r}(t)$ evolves stochastically with time $t$ in a confined domain and ask for the first passage properties of $\mathbf{r}(t)$ to one (or several) targets. A target can represent a critical level to reach before another event is triggered or another molecule with which the walker will react. First passage properties depend on (i) the properties of the stochastic process, often described by the propagator $P\left(\mathbf{r}, t \mid \mathbf{r}_{0}\right)$, which is the probability to be observed at position $\mathbf{r}$ at time $t$ given an initial position $\mathbf{r}_{0}$, and (ii) the geometrical parameters of the problem such as the initial distance to the target, its size, or the size and the shape of the confining domain.

Unfortunately, explicit determinations of FPT are mostly limited to simple geometries such as $1 \mathrm{D}$ and spherically symmetric problems $[1,2]$. The determination of FPT for random walks in realistic confined geometries is not just a theoretical challenge in its own right. It is actually a very relevant issue involved as soon as molecules diffuse in confined media. For example, as biomolecules diffuse through a cell they undergo a series of transformations at precise regions of the cell. An estimate of the time needed to go from one point to another is then an essential step in the understanding of the kinetics of the whole process.

The mean first passage time (MFPT) $\langle\mathbf{T}\rangle$ quantifies the time needed to reach a specific target site, and as such, has clear applications in the context of search processes. It has another interpretation: assume that random walkers are injected at a rate $q$ at position $\mathbf{r}_{0}$ in a confined domain and that these walkers are absorbed at a given target site in the domain. The average number of walkers in the domain in a stationary state is then simply given by $q\langle\mathbf{T}\rangle[12,13]$. The MFPT is a complex quantity, which results from averaging trajectories characterized by a broad range of time scales. Some trajectories reach the target almost directly and contribute to very short time scales whereas other trajectories first hit the domain boundary before reaching the target and can be shown to contribute to much longer time scales [14]. Even if the full distribution of the FPT was derived asymptotically in [14], we will focus on the MFPT in this review.

An important aspect of the problem is the potential existence of memory effects for some random walks, for which the future trajectories are not determined uniquely by the position of the walker at initial time, but depends also on the past trajectory. Such non-Markovian dynamics are found in many examples of particles moving stochastically in complex fluids [15 17] or molecules bound to fluctuating extended structures, as in the model of a tagged monomer of a polymer chain [18], which seems to be observed for example in the motion of chromatin fibers [19 21]. The key question then arises of how the memory properties of the random walk influence the MFPT, and of how to take them into account quantitatively.

In this review, we present important advances in the analysis of FPT statistics that have been obtained in the last decade. Section 2 is devoted to the case of Markovian (memory-less) random walks. We present first a general theory which allows one to accurately evaluate the MFPT for regular random walks in bounded domains. We then briefly mention extensions of 
these results to related first-passage observables such as splitting probabilities and occupation times. We next show that this analytical approach provides a universal scaling dependence of the MFPT on both the volume of the confining domain and the source target distance in the case of general scale-invariant processes. This analysis is applicable to a broad range of stochastic processes characterized by length scale-invariant properties. Section 3 presents an extension of these results to a specific example non-Markovian process, in which a reactive monomer of a Rouse chain looks for a target in a confined domain. We show that the calculation of the MFPT requires the determination of the distribution of the conformation of the full chain at the instant of encounter with the target. Such a theory can be used to derive general asymptotic formulas of the MFPT that take into account the behaviors of the walk at different time scales. Last, section 4 presents the related question of determining the mean cyclization time of a polymer. This problem can in fact be seen as an MFPT problem for a chain confined in an effective volume of diameter of the order of the polymer gyration radius. This problem has been studied intensively and we review briefly the different approaches available in the literature, highlighting the importance of non-Markovian effects.

Let us finally stress that we will consider in this review only MFPTs of random walks in confinement. For a recent review of first-passage processes in absence of confinement, and in particular persistence properties of non-equilibrium processes, the reader is referred to [22].

\section{First-passage observables of Markovian processes}

\subsection{Discrete random walks on regular lattices}

In this first section we consider the case of discrete time random walks on discrete structures, discussed for example in [12]. We introduce both the relevant physical observables (FPTs, occupation times, splitting probabilities) as well as the tools used to determine them (pseudo-Green functions, exact expressions in the case of rectangular domains, large volume asymptotic expansions in the case of arbitrary domains). The formalism is introduced for general graphs, but explicit results are derived in this section for regular Euclidean lattices. The cases of fractal structures and complex networks will be presented in the forthcoming sections. Note that the case of continuous-time random walks (CTRWs) can easily be derived from the formalism introduced in this section. Its analysis can be found in $[23,24]$.

2.1.1. Mean-FPT with a single target. We consider a discrete-time random walk on a discrete lattice of $N$ sites, and we note $w_{x y}$ the probability to jump from site $y$ to site $x$ in one step, which satisfies

$$
\sum_{x} w_{x y}=1
$$

where the sum goes over all the sites $x$ of the lattice. Let $P_{n}(x \mid y)$ be the probability to be at time $n$ at site $x$ knowing that initially the walker was at site $y, F_{n}(x \mid y)$ the probability to visit site $x$ for the fist time at time $n$ knowing that initially the walker was at site $y$ and $P(x)$ the stationary probability to be at site $x$. We first derive an exact expression of the mean firstpassage time $\langle\mathbf{T}(x \mid y)\rangle$ from site $y$ to site $x$ (see figure (1) ) in terms of pseudo-Green functions of the problem, which will be used all along this section. 


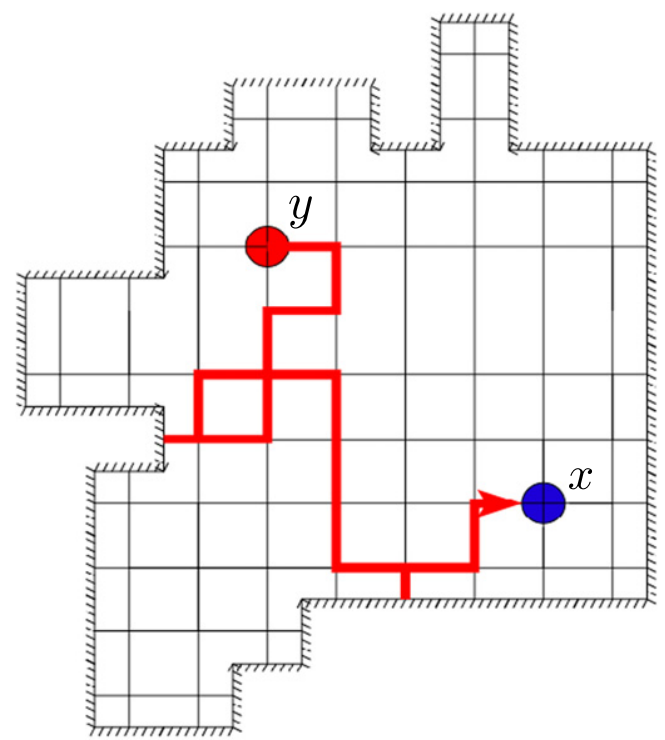

Figure 1. Schematic of the problem. A searcher starts at $y$ and performs a discrete random walk in a confined domain, which contains a target site $x$.

Expression in terms of the pseudo-Green function. The starting point is the renewal equation $[25]$

$$
P_{n}(x \mid y)=\sum_{k=0}^{n} F_{k}(x \mid y) P_{n-k}(x \mid x),
$$

where we have used the usual conventions that $F_{0}(x \mid y)=0$ and $P_{0}(x \mid y)=\delta_{x, y}$ and assumed that $x \neq y$. This equation is obtained by partionning over the first time $k$ the site $x$ is visited, and writing that the walker has to come back at site $x$ in the $n-k$ remaining steps to be still at site $x$ at time $n$. Laplace transforming this temporal convolution equation, we get

$$
\widehat{F}(x, s \mid y)=\frac{\widehat{P}(x, s \mid y)}{\widehat{P}(x, s \mid x)},
$$

where we have introduced the discrete Laplace transforms

$$
\widehat{P}(x, s \mid y) \equiv \sum_{n=0}^{\infty} P_{n}(x \mid y) \mathrm{e}^{-s n} \text { and } \widehat{F}(x, s \mid y) \equiv \sum_{n=0}^{\infty} F_{n}(x \mid y) \mathrm{e}^{-s n} .
$$

It is useful to consider the small Laplace variable $s$ expansions of these quantities:

$$
\begin{aligned}
\widehat{P}(x, s \mid y) & =\sum_{n=0}^{\infty}\left\{P(x)+\left[P_{n}(x \mid y)-P(x)\right]\right\} \mathrm{e}^{-s n} \\
& =\frac{P(x)}{s}+\frac{P(x)}{2}+H_{x y}+o(1), \quad(s \rightarrow 0),
\end{aligned}
$$


where

$$
H_{x y} \equiv \sum_{n=0}^{\infty}\left[P_{n}(x \mid y)-P(x)\right],
$$

and

$$
\widehat{F}(x, s \mid y)=1-s\langle\mathbf{T}(x \mid y)\rangle+o(s) .
$$

Expanding equation (3) at first order in $s$ leads to

$$
\langle\mathbf{T}(x \mid y)\rangle=\frac{1}{P(x)}\left(H_{x x}-H_{x y}\right) .
$$

This equation, which will play a key role in this section, has been obtained by Noh and Rieger in the physics literature [26], and also seems to be known in the mathematics literature (see [27] and references therein). Several comments are in order:

(i) A first important property of the $H$ function resulting directly from its definition (6) and the normalization of $P_{n}(x \mid y)$ and $P(x)$ is that

$$
\sum_{x} H_{x y}=0 .
$$

A second useful property resulting from the detailed balance hypothesis and shown in [24] is that

$$
H_{x y} P(y)=H_{y x} P(x) .
$$

(ii) This $H$ function actually admits a simple physical interpretation. It can be conveniently obtained by first writing a backward equation for $P_{n}$

$$
P_{n+1}(x \mid y)=\sum_{z} w_{z y} P_{n}(x \mid z),
$$

resulting from a partition of the first step of the walk, and the trivial equation

$$
P(x)=\sum_{z} w_{z y} P(x) .
$$

Summing over $n$ the difference of equations (11) and (12) gives:

$$
-\sum_{z} w_{z y}\left(H_{x z}-H_{x y}\right)=\delta_{x y}-P(x),
$$

which indicates that $H$ is actually a pseudo-Green function (see [28]) of the operator involved in the lhs of equation (13), which is a discrete Laplacian. Physically, it corresponds to the stationary solution of a discrete diffusion equation in the presence of a uniformally distributed sink term.

(iii) Expression (8) of the MFPT can be shown using equation (13) to verify the classical backward equation :

$$
-\sum_{z} w_{z y}(\langle\mathbf{T}(x \mid z)\rangle-\langle\mathbf{T}(x \mid y)\rangle)=1
$$

with boundary condition $\langle\mathbf{T}(x \mid x)\rangle=0$.

(iv) The so-called Kac formula [27] is easily derived from this formalism since the mean first return time to site $x$ can be written 


$$
\begin{aligned}
\left\langle\mathbf{T}_{r}(x)\right\rangle & =1+\sum_{z} w_{z x}\langle\mathbf{T}(x \mid z)\rangle \\
& =1+\sum_{z} w_{z x} \frac{H_{x x}-H_{x z}}{P(x)} \\
& =\frac{1}{P(x)},
\end{aligned}
$$

where the first line is a backward equation for the mean return time similar to equation (14), and equations (8) and (13) have been used successively.

(v) The global MFPT [29 31], defined as the MFPT averaged over the initial position of the random walk (with the stationary weight) can be written as:

$$
\begin{aligned}
\overline{\langle\mathbf{T}(x)\rangle} & \equiv \sum_{y}\langle\mathbf{T}(x \mid y)\rangle P(y) \\
& =\frac{1}{P(x)} \sum_{y}\left(H_{x x}-H_{x y}\right) P(y) \\
& =\frac{H_{x x}}{P(x)}-\frac{1}{P(x)} \sum_{y} H_{y x} P(x) \\
& =\frac{H_{x x}}{P(x)}
\end{aligned}
$$

where we have used successively the symmetry relation of the pseudo-Green function (9) and the relation (10).

Finally, we stress that equation (8) is exact and general. However, since it involves the a priori unknown $H$ function, it remains somehow formal at this stage. In the following, we show how explicit expressions of the MFPT can be obtained. Unless otherwise explicitly stated, we will assume that the stationary probability $P(x)$ is uniform

$$
P(x)=\frac{1}{N},
$$

which is the case as soon as the probabilities $w$ are symmetrical (i.e. $w_{x y}=w_{y x}$ for all $x$ and $y$ ). Here, $N$ is the total number of sites that the walker can reach in the graph.

Exact calculation of the MFPT: the case of rectangular domains. In the particular case of a rectangular domain, exact expressions of the pseudo-Green function $H$ can be obtained, both for periodic and reflecting boundary conditions [13, 32]. In turn, using equation (8), this provides exact expressions for the MFPT (and for several other first-passage observables as we will show later). We recall in this section how these exact expressions are obtained in 2D in the case of periodic boundary conditions (see $[13,32]$ for the 3D case and for reflecting boundary conditions).

Let us consider a domain, with $X$ sites in the $x$ direction, $Y$ sites in the $y$ direction. In this case, $w_{\mathbf{r}, \mathbf{r}^{\prime}}=1 / 4$ if $\mathbf{r}$ and $\mathbf{r}^{\prime}$ are nearest neighbors and zero elsewhere. We decompose $H$ into a Fourier sum:

$$
H_{\mathbf{r}, \mathbf{r}^{\prime}}=\sum_{m=0}^{X-1} \sum_{n=0}^{Y-1} a_{m n}\left(\mathbf{r}^{\prime}\right) \exp \left(\frac{2 \mathrm{i} m \pi x}{X}+\frac{2 \mathrm{i} n \pi y}{Y}\right),
$$


where the coordinates of $\mathbf{r}$ are $(x, y, z)$ and the coordinates of $\mathbf{r}^{\prime}$ are $\left(x^{\prime}, y^{\prime}, z^{\prime}\right)$. If we substitute this into equation (13), we obtain an explicit expression of $a_{m n}\left(\mathbf{r}^{\prime}\right)$, which leads to

$$
H_{\mathbf{r}, \mathbf{r}^{\prime}}=\frac{1}{N} \sum_{m=0}^{X-1} \sum_{n=\delta_{m 0}}^{Y-1} \frac{\exp \left(\frac{2 \mathrm{i} m \pi\left(x-x^{\prime}\right)}{X}+\frac{2 \mathrm{i} n \pi\left(y-y^{\prime}\right)}{Y}\right)}{1-\frac{1}{2}\left(\cos \frac{2 m \pi}{X}+\cos \frac{2 n \pi}{Y}\right)} .
$$

Large volume asymptotics of the MFPT. In the case of general confining domains, exact expressions of the pseudo-Green function $H$ are not available. However, large volume asymptotics of the MFPT can be obtained. As we show, they provide accurate expressions even for reasonably small systems and allow us to give first answers to the questions raised above concerning the influence of geometrical parameters on MFPTs.

Actually, even if formal, equation (8) is very well suited to large volume asymptotics of the MFPT. In the following, we consider two prescriptions for this large volume limit: the case when the volume goes to infinity with the constraint that both the target and the starting points of the walk remain infinitely distant from any point of the boundary (target and starting points 'in the bulk'); the case when the volume goes to infinity with the constraint that both the target and the starting points of the walk remain close to a single planar boundary of the domain.

We first consider the case when the target and the starting points are in the bulk.

In this case, (8) leads to

$$
\lim _{N \rightarrow \infty} \frac{\langle\mathbf{T}(x \mid y)\rangle}{N}=\sum_{n=0}^{\infty}\left[P_{n}^{\infty}(x \mid x)-P_{n}^{\infty}(x \mid y)\right],
$$

where $P_{n}^{\infty}(x \mid y)$ stands for the propagator on an infinite lattice. Using standard large distance expressions of these quantities [25], we finally obtain for hypercubic lattices:

$$
\lim _{N \rightarrow \infty} \frac{\langle\mathbf{T}(x \mid y)\rangle}{N}=G_{0}(0)-\frac{\Gamma(d / 2-1)}{2 \pi^{d / 2}} \frac{1}{|x-y|^{d-2}}+o\left(\frac{1}{|x-y|^{d-2}}\right)
$$

if $d>2$, and

$$
\lim _{N \rightarrow \infty} \frac{\langle\mathbf{T}(x \mid y)\rangle}{N}=\frac{2}{\pi} \ln |x-y|+\frac{3}{\pi} \ln 2+\frac{2 \gamma}{\pi}+o(1)
$$

if $d=2$. In these expressions,

$$
G_{0}(x) \equiv \sum_{n=0}^{\infty} P_{n}^{\infty}(x \mid 0)
$$

is the Green function of the unconfined random walk and $\gamma$ is the Euler constant (with $3 / \pi \ln 2+2 \gamma / \pi=1.029374 \ldots)$. Note that in $d=1$, it is found that

$$
\lim _{N \rightarrow \infty} \frac{\langle\mathbf{T}(x \mid y)\rangle}{N}=|y-x|
$$

in agreement with well-known results [2].

Several important conclusions can be reached from these expressions:

(i) In any dimension, the MFPT depends linearly on the volume.

(ii) In dimension $d>2$, the MFPT saturates at large distance $x-y$. In other words, the MFPT loses the memory of the starting point at large separations between the starting and the target points. 


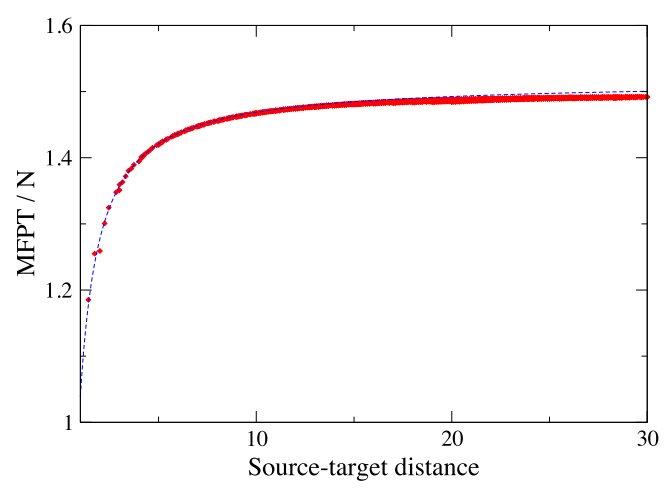

Figure 2. MFPT in 3D parallelepipedic domains: influence of the distance between the source and the target. Red crosses: simulations; blue dashed line: large volume approximation (21). The domain is a cube of side 41, the target being in the middle of it. All the simulation points correspond to different positions of the source.

(iii) The situation is very different in dimensions $d \leqslant 2$, where the MFPT strongly depends on the initial position of the random walker, even at large distances.

As we will show in section 2.2, these conclusions originate from the very nature of the random walk in the absence of any confinement, the so-called type problem. In this context, a random walk is called compact (or recurrent) if it comes back with probability one to its starting point and non-compact (or transient) in the opposite case. The saturation of the MFPT with distance mentioned above is in fact characteristic of non-compact random walks as is the case of $3 \mathrm{D}$ regular random walks, while the strong dependence on the starting position observed for $1 \mathrm{D}$ and $2 \mathrm{D}$ is a hallmark of compact transport. As we will show later, these conclusions will also hold for any Markovian scale-invariant processes (including Lévy flights, random walks on fractal structures ...). Figure 2 displays the accuracy of this large volume approximation even in the case of a small confining cube and illustrates the saturation of the MFPT with the distance mentioned above.

We now consider the situation corresponding to the target and starting points close to a boundary. We assume that the volume goes to infinity with the constraint that both $x$ and $y$ remain close to an infinite reflecting plane $(P)$. In other words, the confining domain tends towards a semi infinite space. The corresponding propagator, denoted by $P_{n}^{\infty / 2}(x \mid y)$ is then easily written in terms of the infinite space propagator $P_{n}^{\infty}(x \mid y)$ by using the method of images [25]:

$$
P_{n}^{\infty / 2}(x \mid y)=P_{n}^{\infty}(x \mid y)+P_{n}^{\infty}(x \mid s(y)),
$$

where $s(y)$ stands for the symmetrical point with respect to the plane $(P)$ of point $y$. Finally, for hypercubic lattices, 


$$
\begin{aligned}
\lim _{N \rightarrow \infty} \frac{\langle\mathbf{T}(x \mid y)\rangle}{N}= & G_{0}(0)-\frac{\Gamma(d / 2-1)}{2 \pi^{d / 2}} \frac{1}{|x-y|^{d-2}} \\
& +\frac{\Gamma(d / 2-1)}{2 \pi^{d / 2}}\left(\frac{1}{|x-s(x)|^{d-2}}-\frac{1}{|x-s(y)|^{d-2}}\right)+\ldots
\end{aligned}
$$

if $d>2$, while

$$
\begin{aligned}
\lim _{N \rightarrow \infty} \frac{\langle\mathbf{T}(x \mid y)\rangle}{N}= & \frac{2}{\pi} \ln |x-y|+\frac{3}{\pi} \ln 2+\frac{2 \gamma}{\pi} \\
& +\frac{2}{\pi} \ln \frac{|x-s(y)|}{|x-s(x)|}+o(1)
\end{aligned}
$$

in $d=2$.

These expressions allow one to discuss several aspects of the influence of the boundaries of the domain.

(i) The qualitative effect of the boundaries is to increase the MFPT when a target is near a boundary, or decrease it when the source is near a boundary.

(ii) The first effect is much more important than the second (because $|x-s(x)|$ is small in the first case, while neither $|x-s(y)|$ nor $|x-s(x)|$ is small in the second one).

(iii) These effects are just as marked when the dimension is small.

2.1.2. Situations with several targets: splitting probabilities. We consider the case when $N$ targets of positions $x_{1}, x_{2}, \ldots, x_{N}$ are present in the problem, and focus on the splitting probability $\pi_{\mathrm{i}}^{x_{1}, \cdots, x_{N}}(y)$ that a random walker starting from site $y$ reaches the target $x_{i}$ before the others. These quantities are especially useful to quantify the kinetics of competitive reactions [4], and may be useful in biology to determine to which extent cellular variability may be controlled by diffusion [33, 34]. It is shown in $[13,35]$ by writing a renewal equation with $N$ targets that the splitting probabilities can be expressed exactly in terms of pseudoGreen functions only. In the particular case of two targets, it is found that

$$
\begin{aligned}
& \pi_{1}^{x_{1}, x_{2}}(y)=\frac{P(1)\left(H_{22}-H_{2 y}\right)+P(2)\left(H_{1 y}-H_{12}\right)}{P(1)\left(H_{22}-H_{21}\right)+P(2)\left(H_{11}-H_{12}\right)}, \\
& \pi_{2}^{x_{1}, x_{2}}(y)=\frac{P(2)\left(H_{11}-H_{1 y}\right)+P(1)\left(H_{2 y}-H_{21}\right)}{P(1)\left(H_{22}-H_{21}\right)+P(2)\left(H_{11}-H_{12}\right)}
\end{aligned}
$$

where we have used the notations $H_{i j} \equiv H_{x_{i} x_{j}}$. As in the case of MFPTs with a single target presented in section 2.1.1), exact expressions are obtained in the case of rectangular domains, and large volume asymptotics can be derived in the general case. These two-target results are displayed in figure 3 , which shows that the accuracy of the large volume asymptotic expressions even for finite domains.

An important qualitative difference between the 2D and 3D cases is revealed by the large volume asymptotics of the pseudo-Green function. In 3D, the furthest target always has a significant probability to be reached first, since the most important terms in the probabilities $P_{i}$ are $H_{11}$ and $H_{22}$. In 2D, if a target is much closer from the source than the other, it will almost certainly be reached first, since $H_{\mathbf{r}_{i}, \mathbf{r}_{j}}$ scales as $\ln \left|\mathbf{r}_{i}-\mathbf{r}_{j}\right|$. Actually, the probability for the furthest target to be reached first decreases logarithmically. As will be discussed on more general grounds in section 2.2, these properties are related to the non-compact character of the 


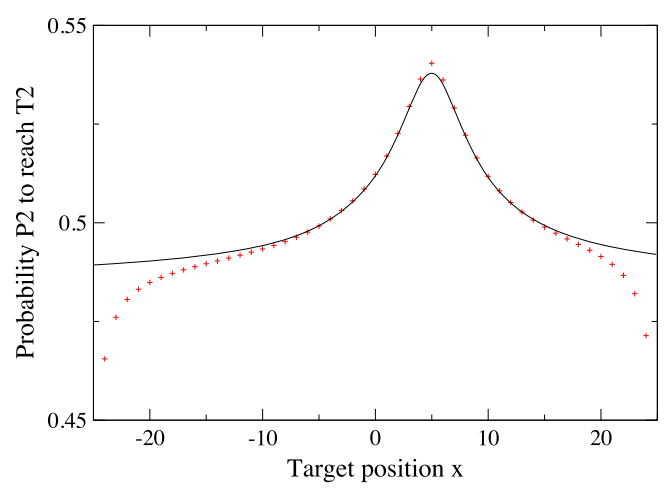

Figure 3. Splitting probability in 3D domains. Simulations (red crosses) versus theory with the large volume approximation of the pseudo Green function $H=G_{0}$ (solid line). One target is fixed in $(-5,0,0)$; the source is fixed in $(5,0,0)$; the other target is at $(\mathrm{x}$, $3,0)$. The domain is a cube of side 51 , the middle is the point $(0,0,0)$.

infinite 3D walk, and the compact character of the 2D walk. Indeed, an infinite 2D walk explores all the sites of the lattice, whereas an infinite 3D walk does not; we may thus consider that the 2D walk will explore most of the sites surrounding the source before going much further, whereas the 3D walk will not, which qualitatively explains the difference of behaviour. This behavior parallels the dependance of the MFPT on the starting distance described above.

2.1.3. Occupation times. We now consider the time $N^{(x)}(z \mid y)$ spent on a given site $z$ by a random walker starting from site $y$ before reaching a target $x$. The study of the statistics of this general quantity, known in the random walk literature as the occupation time, has long been a subject of interest, both for mathematicians [25, 27] and physicists [36 46]. As a matter of fact, the occupation time has proven to be a key quantity in various fields, ranging from astrophysics [47], transport in porous media [48] and diffusion-limited reactions [49]. The point is that as soon as some specific sites of a system have different physical or chemical properties, it becomes crucial to know precisely how many times each site is visited by the random walker.

As the MFPT, the mean occupation time can easily been written in terms of the pseudoGreen functions [50]:

$$
\left\langle N^{(x)}(z \mid y)\right\rangle=\frac{P(z)}{P(x)}\left(H_{x x}-H_{x y}\right)+H_{z y}-H_{z x}
$$

Note that this result also gives the mean occupation time of a subdomain, which is simply the sum of the mean occupation time of all the sites in the subdomain. In particular, using equation (9), we can check that the mean occupation time for the whole domain,

$$
\sum_{z}\left\langle N^{(x)}(z \mid y)\right\rangle=\frac{1}{P(x)}\left(H_{x x}-H_{x y}\right)
$$

gives back the MFPT from $y$ to $z$ provided by equation (8). The information provided by the mean occupation is therefore finer than the MFPT. 
2.1.4. Extension to continuous Brownian motion. The expressions obtained earlier in the case of discrete time and space random walks can be extended to the case of continuous time and space Brownian motion. We therefore now consider a continuous Brownian motion of diffusion coefficient $D$ in a confining domain with reflecting boundary conditions, and we still want to know how long it takes to reach a given target. A fundamental difference with the discrete case is that, except in the 1D case, the target must have a finite size to be reached, and this size $a$ will be an important parameter of the problem. This question has also been addressed in the mathematical literature (see for instance [51 54]).

In fact, taking the limit of small target size makes it possible to directly transpose essentially all the results on discrete random walks obtained in the previous section $[13,55]$. More precisely, in the small target size limit, the expressions of the MFPT and splitting probabilities obtained in the discrete case hold also in this continuous case by making use of

$$
H\left(\mathbf{r}_{T} \mid \mathbf{r}_{T}\right) \equiv G_{0}(a)+H^{*}\left(\mathbf{r}_{T} \mid \mathbf{r}_{T}\right)
$$

where $H^{*}\left(\mathbf{r} \mid \mathbf{r}_{T}\right)$ is defined as the regular part of $H\left(\mathbf{r} \mid \mathbf{r}_{T}\right)$ as $\mathbf{r} \rightarrow \mathbf{r}_{T}$ :

$$
H^{*}\left(\mathbf{r} \mid \mathbf{r}_{T}\right)=H\left(\mathbf{r} \mid \mathbf{r}_{T}\right)-G_{0}\left(\mathbf{r}-\mathbf{r}_{T}\right),
$$

and $G_{0}\left(\mathbf{r}-\mathbf{r}_{T}\right)$ is the infinite space Green function.

As in the discrete case, the pseudo-Green function $H$ is known exactly for domains with simple shapes such as spheres. For example the pseudo-Green function for the unit disk of surface area $A=\pi$ reads [28]:

$$
H\left(\mathbf{r} \mid \mathbf{r}^{\prime}\right)=\frac{1}{2 \pi}\left[-\ln \left|\mathbf{r}-\mathbf{r}^{\prime}\right|-\ln B\left(\mathbf{r}, \mathbf{r}^{\prime}\right)+\frac{1}{2}\left(r^{2}+r^{\prime 2}\right)-\frac{3}{4}\right],
$$

where $B$ is the modulus of

$$
\mathbf{B}=r^{\prime} \mathbf{r}-\frac{\mathbf{r}^{\prime}}{r^{\prime}}
$$

Figure 4 shows the accuracy of the method on the example of a 2D disk with an off-centered target.

For a domain of arbitrary shape, there is no explicit analytical expression of the pseudoGreen function. However, as in the discrete case, large volume asymptotics of first-passage observables can be derived. Indeed, one has to leading order $\mathrm{H} \approx G_{0}$ where $G_{0}$ is the infinite space Green function. This leads to explicit expressions of first-passage observables in terms of $G_{0}$ only (see $[13,56]$ ). For example, the MFPT to a single target starting from $S$ writes:

$$
\begin{aligned}
& \lim _{V \rightarrow \infty} \frac{\left\langle\mathbf{T}\left(\mathbf{r}_{S}\right)\right\rangle}{V}=\frac{1}{4 \pi D}\left(\frac{1}{a}-\frac{1}{r}\right) \\
& \lim _{A \rightarrow \infty} \frac{\left\langle\mathbf{T}\left(\mathbf{r}_{S}\right)\right\rangle}{A}=\frac{1}{2 \pi D} \ln \frac{r}{a} \quad(2 \mathrm{D})
\end{aligned}
$$

$r$ being the source target distance.

2.1.5. Alternative approach: renewal equation for continuous processes. The results presented in the previous section for continuous processes can in fact be derived directly from a renewal equation, as we show in this paragraph. This alternative approach will prove to be useful in the case of non-Markovian processes. We assume that the stochastic process can be defined by the propagator $P\left(\mathbf{r}, t \mid \mathbf{r}_{0}\right)$ (in confined space), which gives the probability density to observe the walker at $\mathbf{r}$ at time $t$, given that the initial position was $\mathbf{r}_{0}$. Defining the 


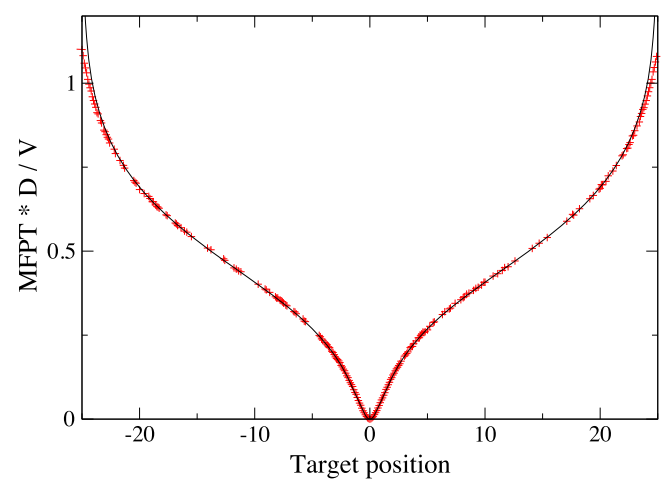

Figure 4. Brownian motion on a $2 \mathrm{D}$ disk of radius 25 centered at $(0,0)$; the source is at $(0,1)$ and the target of radius 1 is at $(x, 0)$. Red crosses: simulations; black solid line: theoretical prediction of equation (8) with the substitution of equation (32) where the exact function $H$ given by equation (34).

target as the sphere of radius $a$ and center $\mathbf{r}=\mathbf{0}$, and $f\left(t, \mathbf{r}^{\prime} \mid \mathbf{r}_{S}\right)$ as the FPT density at the target at $\mathbf{r}^{\prime}$ starting from $\mathbf{r}_{S}$, one can write the following renewal equation:

$$
P\left(\mathbf{r}, t \mid \mathbf{r}_{S}\right)=\int_{0}^{t} \mathrm{~d} t^{\prime} \int_{\left|\mathbf{r}^{\prime}\right|=a} \mathrm{~d} S\left(\mathbf{r}^{\prime}\right) f\left(t^{\prime}, \mathbf{r}^{\prime} \mid \mathbf{r}_{S}\right) P\left(\mathbf{r}, t \mid \mathbf{r}^{\prime}\right)
$$

where $\mathbf{r}$ can be any point inside the target. Taking $\mathbf{r}=\mathbf{0}$, and expanding the Laplace transform of (38) for small values of the Laplace variable, we get:

$\left\langle\mathbf{T}\left(\mathbf{r}_{S}\right)\right\rangle V^{-1}=\int_{0}^{\infty} \mathrm{d} t \int_{\left|\mathbf{r}^{\prime}\right|=a} \mathrm{~d} S\left(\mathbf{r}^{\prime}\right)\left\{P\left(\mathbf{0}, t \mid \mathbf{r}^{\prime}\right) \pi\left(\mathbf{r}^{\prime} \mid \mathbf{r}_{S}\right)-P\left(\mathbf{0}, t \mid \mathbf{r}_{S}\right)\right\}$

where $\pi\left(\mathbf{r}^{\prime} \mid \mathbf{r}_{S}\right)=\int_{0}^{\infty} \mathrm{dt} f\left(t, \mathbf{r}^{\prime} \mid \mathbf{r}_{S}\right)$ is the splitting probability to arrive on the target surface at position $\mathbf{r}^{\prime}$. We next introduce the infinite space propagator $P_{\infty}\left(\mathbf{r}, t \mid \mathbf{r}_{0}\right)$, which we assume depends only on the distance $\left|\mathbf{r}-\mathbf{r}_{0}\right|$. Averaging (39) over $\mathbf{r}_{S}$ and taking the large volume limit then yields

$$
\lim _{V \rightarrow \infty} \frac{\langle\mathbf{T}\rangle}{V}=\int_{0}^{\infty} \mathrm{d} t\left\{P_{\infty}\left(\mathbf{0}, t \mid a \mathbf{u}_{r}\right)-P_{\infty}\left(\mathbf{0}, t \mid \mathbf{r}_{0}\right)\right\}
$$

where $\mathbf{u}_{r}$ is a unit vector pointing normal to the target center. This formula relates explicitly the propagator in infinite space, $P_{\infty}$, to the mean first passage time. For example, in the case of Brownian diffusion, the propagator is known explicitly,

$$
P_{\infty}\left(\mathbf{r}, t \mid \mathbf{r}_{0}\right)=\frac{1}{(4 \pi D t)^{d / 2}} \mathrm{e}^{-\left(\mathbf{r}-\mathbf{r}_{0}\right)^{2} / 4 D t},
$$

which, once inserted into (40), leads to equations (36), (37).

\subsection{General scale-invariant processes}

So far we have presented general results on first-passage observables valid for regular diffusion processes, either discrete or continuous. We now show that such results can be generalized to the much wider class of scale-invariant processes that include Lévy flights, random walks on fractals and in disordered media (see figure (5)). Note that these results can be straightforwardly generalized to CTRWs on fractals [24]. In this section we present results for 

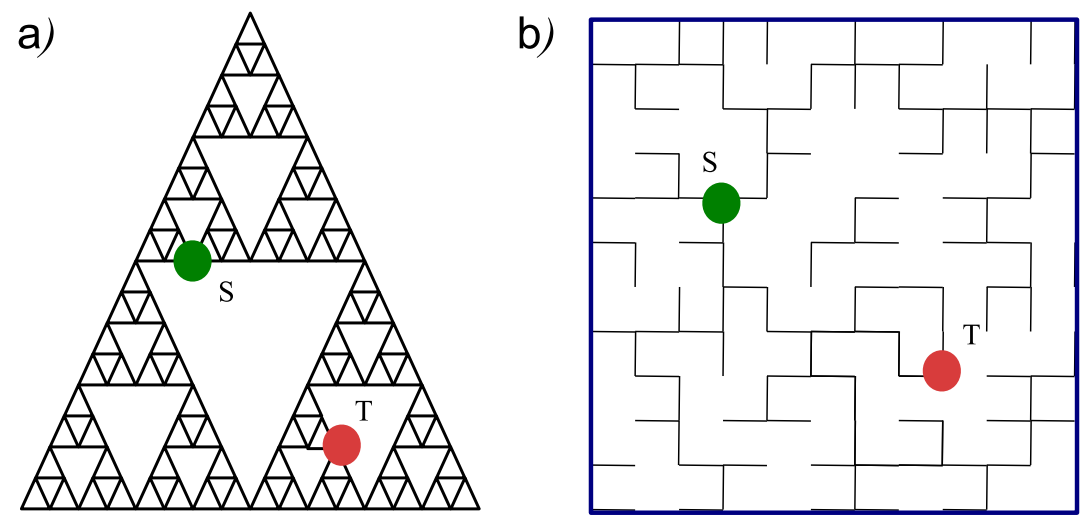

Figure 5. Examples of bounded fractal domains. The random walker starts from $\mathrm{S}$ and reaches T. (a) Sierpinski gasket (here of order 5). (b) Two dimensional critical percolation cluster (case of bond percolation on a square lattice) in a bounded domain.

the first-passage observables introduced earlier: FPTs, splitting probabilities and occupation times. Technically, we derive general large volume asymptotics, by directly paralleling the approach introduced in the case of regular diffusion processes. These results in fact provide explicitly the dependence of these observables on the geometrical (volume and source to target distance) and dynamical (fractal and walk dimension to be defined below) parameters.

2.2.1. MFPT. As in section 2.1, we consider a Markovian random walker moving in a bounded domain of $N$ sites, with reflecting walls. Let $P_{n}(x \mid y)$ be the propagator at time $n$, starting from the site $y$ at time 0 . For the sake of simplicity we assume that the walker performs symmetric jumps and that the stationary distribution is homogeneous $\lim _{n \rightarrow \infty} P_{n}(x \mid y)=P(x)=1 / N$. For an analysis of non-homogeneous networks see for instance [57]. We start from the exact expression for the MFPT derived earlier (see section 2.1), which in fact only requires that the processes is Markovian:

$$
\langle\mathbf{T}(x \mid y)\rangle=\frac{1}{P(x)}\left(H_{x x}-H_{x y}\right) .
$$

Here again, $H$ is the pseudo-Green function [58] of the domain:

$$
H_{x y} \equiv \sum_{n=0}^{\infty}\left(P_{n}(x \mid y)-P(x)\right) .
$$

As in section 2.1.1, we take the large volume limit which yields:

$$
\lim _{N \rightarrow \infty} \frac{\langle\mathbf{T}(x \mid y)\rangle}{N}=\sum_{n=0}^{\infty}\left[P_{n}^{\infty}(x \mid x)-P_{n}^{\infty}(x \mid y)\right],
$$

where $P_{n}^{\infty}(x \mid y)$ stands for the propagator on an infinite lattice.

To go further and obtain explicit results, we follow [59] and assume that the problem is scale-invariant. More precisely, we use for $P^{\infty}$ the standard scaling [60]:

$$
P_{n}^{\infty}(x \mid y) \sim n^{-d_{f} / d_{w}} \Pi\left(\frac{|x-y|}{n^{1 / d_{w}}}\right),
$$


where the fractal dimension $d_{f}$ characterizes the accessible volume $V_{r} \sim r_{f}^{d}$ within a sphere of radius $r$, and the walk dimension $d_{w}$ characterizes the distance $r \sim n^{1 / d_{w}}$ covered by a random walker in a given time $n$. The form (45) ensures the normalization of $P^{\infty}$ by integration over the whole fractal set. Note that the MSD is then given by $\left\langle\Delta \mathbf{r}^{2}\right\rangle \sim n^{\beta}$ with $\beta=2 / d_{w}$. A derivation given in [59] then yields for the MFPT:

$$
\langle\mathbf{T}\rangle \sim \begin{cases}N\left(A-B r^{d_{w}-d_{f}}\right) & \text { for } d_{w}<d_{f} \\ N(A+B \ln r) & \text { for } d_{w}=d_{f}, \\ N\left(A+B r^{d_{w}-d_{f}}\right) & \text { for } d_{w}>d_{f}\end{cases}
$$

where explicit expressions of $A$ and $B$ are given in [59]. This result generalizes the asymptotics derived in the case of regular random walks $\left(d_{w}=2\right)$ on Euclidean lattices of dimension $d_{f}=d$ discussed in section 2.1.

Several comments are in order, which complement the analysis of section 2.1. First, we point out that equation (46) gives the large $N$ asymptotics of the MFPT as a function of $N$ and $r$ as independent variables. In particular the volume dependence is linear with $N$ for $r$ fixed in any case, which can not be inferred from the standard scaling $\langle\mathbf{T}\rangle \propto L^{d_{w}}, L$ being the characteristic length of the domain of order $N^{1 / d_{f}}$. However, a global rescaling of the problem $r \rightarrow \lambda r, L \rightarrow \lambda L$, when applied to equation (46), gives the standard form $\langle\mathbf{T}\rangle \sim \lambda^{d_{w}}$ for $d_{w}>d_{f}$ and $\langle\mathbf{T}\rangle \sim \lambda^{d_{f}}$ for $d_{w}<d_{f}$ in accord with [61, 62]. Second, equation (46) shows two regimes, which rely on infinite space properties of the walk: in the case of compact exploration [60] $\left(d_{w} \geqslant d_{f}\right)$ where each site is eventually visited, the MFPT behaves like $\langle\mathbf{T}\rangle \propto N r^{d_{w}-d_{f}}\left(\langle\mathbf{T}\rangle \propto N \ln r\right.$ for $\left.d_{w}=d_{f}\right)$ at a large distance, so that the dependence on the starting point always matters; inthe opposite case of non-compact exploration $\langle\mathbf{T}\rangle$ tends to a finite value for large $r$, and the dependence on the starting point is lost.

These analytical results can be confirmed by Monte Carlo simulations and exact enumeration methods applied to various models which cover the three previous cases [59, 63].

- The random barrier model in two dimensions [60] is a widespread model of transport in disordered systems whose MFPT properties remain widely unexplored. It is defined by a lattice random walk with nearest-neighbor symmetrical transition rates $\Gamma$ distributed according to some distribution $\rho(\Gamma)$. Even for a power law distribution $\rho(\Gamma)$ the scaling function $\Pi(\xi)$ can be shown to be Gaussian [48] $\left(d_{f}=d_{w}=2\right)$, which allows us to explicitely compute the constant $B$ and obtain $\langle\mathbf{T}\rangle \sim N\left(A+\left(1 / 2 \pi D_{\text {eff }}\right) \ln r\right)$. Here $D_{\text {eff }}$ is a diffusion constant depending on $\rho(\Gamma)$ which can be determined by an effective medium approximation [48, 59].

- The Sierpinski gasket [60] of finite order is a representative example of a deterministic fractal. In this case $d_{f}=\ln 3 / \ln 2<\ln 5 / \ln 2=d_{w}$, so that the theory predicts the scaling $\langle\mathbf{T}\rangle \sim N r^{(\ln 5-\ln 3) / \ln 2}$.

- The Lévy flight model of anomalous diffusion [25, 64, 65] is based on a fat-tailed distribution of jump lengths $p(l) \propto l^{-d-\beta}(0<\beta \leqslant 2)$. The walk dimension is now $d_{w}=\beta$, while the fractal dimension is the dimension of the Euclidean space $d$. In 
dimensions $d \geqslant 2$, or in $1 \mathrm{D}$ when $\beta<1$, one has $d_{f}>d_{w}$ and the theory gives $\langle\mathbf{T}\rangle \sim N\left(A-B r^{\beta-d}\right)$.

- Critical percolation clusters (see figure 1(b)) constitute a representative example of random fractals $[60,66,67]$. Here we consider the case of bond percolation, where the bonds connecting the sites of a regular lattice of the $d$ dimensional space are present with probability $p$. The ensemble of points connected by bonds is called a cluster. If $p$ is above the percolation threshold $p_{c}$, an infinite cluster exists. If $p=p_{c}$, this infinite cluster is a random fractal characterized by its fractal dimension $d_{f}$. We consider a nearest neighbor random walk on such critical percolation cluster with the so-called 'blind ant' [25] dynamics: on arrival at a given site $\mathbf{s}$, the walker attempts to move to one of the adjacent sites on the original lattice with equal probability. If the link corresponding to this move does not exist, the walker remains at site $\mathbf{s}$. This walk is characterized by the walk dimension $d_{w}$. In the example of the 3-dimensional cubic lattice, one has $d_{f}=2.58 \ldots$, and $d_{w}=3.88 \ldots$ [67] and the motion is subdiffusive with $\beta=2 / d_{w} \simeq 0.51 \ldots$

Such models have been frequently used to describe transport processes in real complex media, for example: in the case of exciton trapping on percolation systems [68] or in the case of anomalous motions induced by obstruction and binding in crowded environments $[65,69,70]$ such as biological cells $[63,7174]$. In particular, fractal models have regained interest in the context of the nuclear organization of DNA [75]. Indeed, recent experiments based on neutron scattering [76], rheology techniques [77] and more recently the Hi-C method [78] revealed independently a fractal structure of the chromatin.

Figure 6 reveals an excellent quantitative agreement between the analytical predictions and the numerical simulations. Both the volume dependence and the source target distance dependence are unambiguously captured by the theoretical expressions (46) as shown by the data collapse of the numerical simulations. We emphasize that the very different nature of these examples demonstrates that the range of applicability of the approach, which mainly relies on the length scale-invariant property of the infinite space propagator (45), is wide.

These results can be partly reproduced by using other methods such as electrical analogies $[79,80]$ and elastic vibrations spectrum analysis [81]. It has also been extended in several directions. First, in the case where the scaling form of equation (45) holds at all scales it is shown in [82] that in the compact case $A=0$. Approximations of the constant $B$, independent of the scaling function $\Pi$ can also be obtained by using the Kac formula. This yields in the compact case a zero constant formula for the MFPT, which has been shown to be in very good agreement with numerical results in various examples [82]. Note that these results rely on the scale invariance hypothesis; examples of anisotropic media have been studied in [80]. Finally, we note that, in the case of deterministic fractals, the scaling relation equation (45) actually only holds for discrete rescaling of the distance $|x-y|$. This can be shown to induce small amplitude oscillations of the MFPT with the source to target distance, which can be analytically derived by adapting the above formalism [83].

2.2.2. Further first-passage observables. Following the methods developed in section 2.1, the explicit geometric dependence of further first-passage observables of scale-invariant Markovian processes can be derived. Indeed, the large volume asymptotics of the exact expressions of first-passage observables in terms of pseudo-Green functions derived earlier in section 2.1 are straightforwardly obtained by making the substitution $H(x \mid y) \rightarrow G_{0}(x \mid y) \equiv$ 

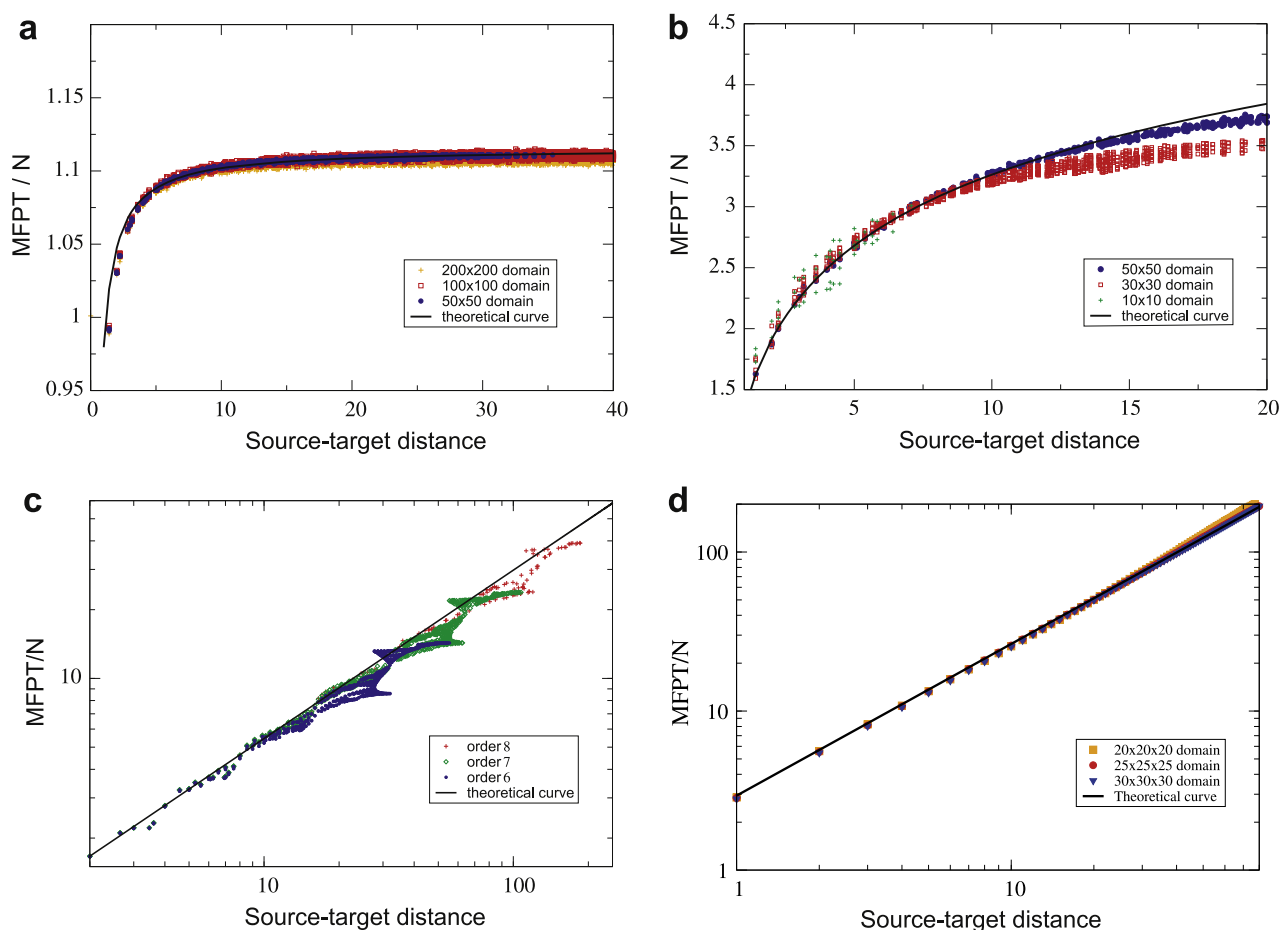

Figure 6. MFPT in scale invariant media. a Lévy flights on a 2D square lattice $(\beta=1)$. The confining domains are (a) $50 \times 50,100 \times 100$ and $200 \times 200$ squares, with the target approximately in the middle. The MFPT is presented as a function of the source target distance for different source points. Simulation points are fitted with $\langle\mathbf{T}\rangle \sim N\left(A-B r^{\beta-2}\right)$. (b) Random barrier models with a transition rate distribution $\rho(\Gamma)=(\alpha / \Gamma)\left(\Gamma / \Gamma_{0}\right)^{\alpha}$, with $\Gamma_{0}=1$ and $\alpha=0.5$. The confining domain is a $L \times L$ square with the target point in the middle. Numerical simulations of the MFPT rescaled by the volume $N$, averaged over the disorder, for three different domain sizes. The theoretical curve (black line) is given by $\langle\mathbf{T}\rangle / N \sim\left(A+\left(1 / 2 \pi D_{\text {eff }}\right) \ln r\right)$, where the only fitting parameter is $A$. (c) Numerical simulations of random walks on a Sierpinski gasket (log/log plot) for three different system sizes (order 6, 7 and 8). For each set of points, the size of the Sierpinski gasket and the target point are fixed and the starting point takes various positions on the Sierpinski gasket. The plain line corresponds to the theoretical scaling $r^{d_{w}-d_{f}}$. (d) $3 \mathrm{D}$ critical percolation clusters. For each size of the confining domain, the MFPT, normalized by the number of sites $\mathrm{N}$, is averaged both over the different target and starting points separated by the corresponding chemical distance, and over percolation clusters. The plain black curve corresponds to the theoretical prediction of equation (46) with $d_{w}-d_{f} \simeq 1$.

$\sum_{n=1}^{\infty} P_{n}^{\infty}(x \mid y)$ and using equation (45). Note that only differences of $H$ functions are involved, which always remain finite in the large volume limit.

For example, it is possible to compute splitting probabilities within this framework. We consider here two targets located at $x_{1}$ and $x_{2}$ and a source point $y$. The probability $\pi_{1}$ of first reaching target 1 , starting from $y$, is then [63]: 


$$
\pi_{1} \sim \begin{cases}\frac{A+B\left(r_{1 y}^{d_{w}-d_{f}}-r_{2 y}^{d_{w}-d_{f}}-r_{12}^{d_{w}-d_{f}}\right)}{2\left(A-B r_{12}^{d_{w}-d_{f}}\right)} & \text { for } d_{w}<d_{f} \\ \frac{A+B \ln \left(r_{2 y} r_{12} / r_{1 y}\right)}{2\left(A+B \ln \left(r_{12}\right)\right)} & \text { for } d_{w}=d_{f} \\ \frac{1}{2}\left(\left(r_{2 y} / r_{12}\right)^{d_{w}-d_{f}}-\left(r_{1 y} / r_{12}\right)^{d_{w}-d_{f}}+1\right) & \text { for } d_{w}>d_{f}\end{cases}
$$

where $r_{i j}=\left|\mathbf{r}_{i}-\mathbf{r}_{j}\right|$ is different from 0 . The mean occupation time of site $i$ starting form site $S$ before reaching site $T$ can also be calculated [63], and reads:

$$
\left\langle\mathbf{N}_{\mathbf{i}}\right\rangle \sim \begin{cases}A+B\left(r_{i S}^{d_{w}-d_{f}}-r_{i T}^{d_{w}-d_{f}}-r_{S T}^{d_{w}-d_{f}}\right) & \text { for } d_{w}<d_{f} \\ A+B \ln \left(r_{i T} r_{S T} / r_{i S}\right) & \text { for } d_{w}=d_{f}, \\ B\left(r_{i T}^{d_{w}-d_{f}}+r_{S T}^{d_{w}-d_{f}}-r_{i S}^{d_{w}-d_{f}}\right) & \text { for } d_{w}>d_{f}\end{cases}
$$

Note that the entire distribution of $\mathbf{N}_{\mathbf{i}}$, which involves only splitting probabilities, can be derived [63].

\section{Mean first-passage times of non-Markovian processes in confinement: the example of the Rouse chain}

\subsection{MFPT for non-Markovian processes and the Markovian approximation}

Up to now, all the theories for the MFPT that we have considered make an explicit use of the Markovian properties of the stochastic processes at the very beginning of the analysis. For example, the expression (8) states that the MFPT can be expressed as time integrals of the propagator of the process, which is ill defined for generic processes with memory. The case of non-Markovian processes therefore requires a further analysis that we present here. We consider now a stochastic process $\mathbf{X}(t)$ evolving with continuous time $t$ with stationary increments. As stated in [84], the non-Markovian character of a process often arises from the interaction of the considered variable, $\mathbf{X}(t)$, with other degrees of freedom that evolve at a time scale comparable to that of the motion. A typical example of non-Markovian walker is the motion of one tagged monomer of a polymer chain [85], or of one tagged particle moving in a complex fluid [15 17,86]. Here, specifically, we consider the overdamped dynamics of $N$ particles of positions $\mathbf{x}_{1}, \ldots, \mathbf{x}_{N}$ that evolve in a space of dimension $d$ and interact through harmonic interactions and hence satisfy the Fokker Planck equation:

$$
\frac{\partial P}{\partial t}=\sum_{i, j=1}^{N} \nabla_{i} A_{i j} \mathbf{x}_{j} P+D \sum_{i=1}^{N} \nabla_{i}^{2} P,
$$

where the connectivity matrix $A$ is symmetric, and $D$ is the individual diffusion coefficient of each particle. The case of a flexible (Rouse) chain [87], where a polymer is simply described by a chain of beads with friction coefficient $\zeta$ linked by springs of stiffness $k$, is obtained when $A$ takes the tridiagonal form: 


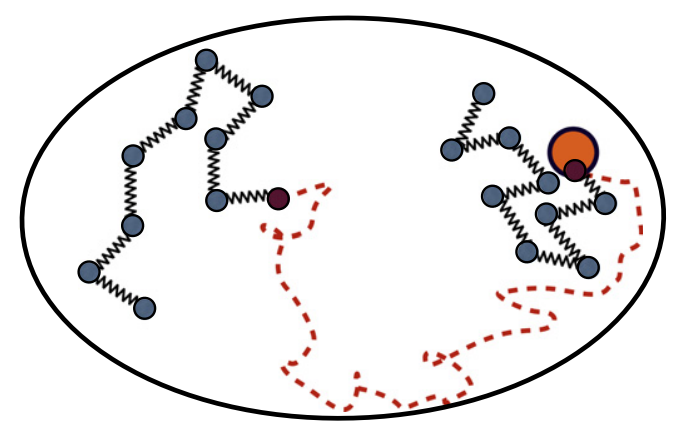

Figure 7. Schematic of the MFPT problem in the non Markovian case: a monomer of a Rouse chain is searching for a target in a confined domain.

$$
A=\frac{k}{\zeta}\left(\begin{array}{cccccc}
1 & -1 & 0 & . . & . . & . . \\
-1 & 2 & -1 & 0 & . . & . . \\
0 & -1 & 2 & -1 & . . & . . \\
. . & . . & . . & . & . . & . \\
. . & . . & 0 & -1 & 2 & -1 \\
. . & . . & . . & 0 & -1 & 1
\end{array}\right) .
$$

We now assume that the non-Markovian walker $\mathbf{X}(t)$ is one of the monomers, called below a reactive monomer, at position $q$ in the the chain, $\mathbf{X}(t)=\mathbf{x}_{q}(t)$, and we study the MFPT of $\mathbf{X}(t)$ to a fixed target (see figure 7). We furthermore assume that the polymer is confined inside a volume $V$ much larger than its gyration radius, and that both the initial position $\mathbf{X}(t=0)$ and the target position are in the bulk, that is far from the confining boundaries. The Rouse model will be our main example of non-Markovian process. However, depending on the choice of matrix $A$, the models of the type equation (49) can be used to describe more complicated structures, such as Gaussian semiflexible chains [88] (in which successive bonds tend to be aligned), chains with dihedral interactions [89], the dynamics of interfaces [90], etc, and the theory described here does not depend on the precise shape of the matrix $A$. In the following, we describe the essential steps to obtain a precise estimate of the MFPT in this non-Markovian problem.

The equation (49) is generally simplified by using normal mode analysis. We define the orthogonal matrix $Q$ such that $A=Q \operatorname{diag}\left(\lambda_{1}, \ldots, \lambda_{N}\right) Q^{-1}$, with $\operatorname{diag}\left(\lambda_{1}, \ldots, \lambda_{N}\right)$ a diagonal matrix of eigenvalues $\lambda_{i}$, with $\lambda_{1}=0<\lambda_{2}<\ldots<\lambda_{N}$. The modes $\mathbf{a}_{i}$ are defined by:

$$
\mathbf{a}_{i}(t)=\sum_{j=1}^{N} Q_{i j} \mathbf{x}_{j}(t), \quad \mathbf{x}_{i}(t)=\sum_{j=1}^{N} Q_{j i} \mathbf{a}_{j}(t) .
$$

The evolution of the probability density $P(\{\mathbf{a}\}, t)$ to observe the system with a configuration $\{\mathbf{a}\}=\left\{\mathbf{a}_{1}, \ldots, \mathbf{a}_{N}\right\}$ at time $t$ in the absence of absorption now satisfies the simplified Fokker Planck equation:

$$
\frac{\partial P}{\partial t}=\sum_{i=1}^{N} \frac{\partial}{\partial \mathbf{a}_{i}}\left(\lambda_{i} \mathbf{a}_{i} P+D \frac{\partial}{\partial \mathbf{a}_{i}} P\right),
$$

where each mode evolves independently of the others. In terms of the modes, the variable $\mathbf{X}$ is expressed as the linear combination: 


$$
\mathbf{X}(t)=\sum_{i=1}^{N} b_{i} \mathbf{a}_{i}(t), \quad b_{i} \equiv Q_{q i} .
$$

We study the problem of finding the MFPT of $\mathbf{X}(t)$ to a spherical target of radius $a$ located around the position $\mathbf{X}=\mathbf{0}$. For simplicity, we restrict our analysis to initial configurations such that $\mathbf{X}=\mathbf{X}_{0}$ and such that the rest of the polymer is at equilibrium:

$$
P_{\text {ini }}(\{\mathbf{a}\})=\left|b_{1}\right|^{d}\left[\frac{\lambda_{2} \ldots \lambda_{N}}{(2 \pi D)^{N-1}}\right]^{d / 2} \exp \left(-\sum_{i=2}^{N} \frac{\lambda_{i} \mathbf{a}_{i}^{2}}{2 D}\right) \delta\left(\sum_{i=1}^{N} b_{i} \mathbf{a}_{i}-\mathbf{X}_{0}\right) \text {. }
$$

The above distribution is Gaussian, with mean vector $\left\langle\mathbf{a}_{i}\right\rangle_{\mathrm{ini}}=\delta_{i, 1} \mathbf{X}_{0} / b_{1}$, and covariance matrix $\operatorname{Cov}\left(a_{i \alpha}, a_{j \beta}\right)=\sigma_{i j}^{\mathrm{eq}, *} \delta_{\alpha \beta}$ with $\alpha, \beta$ the spatial coordinates $\{x, y, z\}$, and $\sigma_{i j}^{\text {eq,** }}$ is defined by:

$$
\sigma_{i j}^{\mathrm{eq}, *}=D \begin{cases}\delta_{i j} / \lambda_{i} & \text { if } i, j \geqslant 2 \\ -b_{j} /\left(b_{1} \lambda_{j}\right) & \text { if } j \geqslant 2, i=1 . \\ \sum_{q=2}^{N} b_{q}^{2} /\left(\lambda_{q} b_{1}^{2}\right) & \text { if } i=j=1\end{cases}
$$

Note that $\sum_{j} \sigma_{i j}^{\mathrm{eq}, *} b_{j}=0$, which imposes $\mathbf{X}=\mathbf{X}_{0}$ at $t=0$, as seen by the presence of the delta function in equation (54). Interestingly, $\sigma_{i j}^{\text {eq,** }}$ does not depend on the value of $\mathbf{X}_{0}$. Now, let $\delta_{\alpha \beta} \gamma_{i j}(t)$ be the covariance of $a_{i \alpha}$ and $a_{j \beta}$ at a time $t$ after the initial time in unbounded space and in the absence of target. It is known [84] that the Fokker Planck equation (52) admits Gaussian solutions and that the evolution of the covariance matrix $\gamma_{i j}$ satisfies the following equation:

$$
\partial_{t} \gamma_{i j}=-\left(\lambda_{i}+\lambda_{j}\right) \gamma_{i j}+2 D \delta_{i j}
$$

The actual value of $\gamma_{i j}(t)$ is found by solving equation (56) with the initial condition $\gamma_{i j}(0)=\sigma_{i j}^{\text {eq,** }}$ given by equation (55):

$$
\gamma_{i j}(t)=\delta_{i j} D\left(1-\mathrm{e}^{-2 \lambda_{i} t}\right) / \lambda_{i}+\mathrm{e}^{-\lambda_{i} t} \mathrm{e}^{-\lambda_{j} t} \sigma_{i j}^{\mathrm{eq}, *}
$$

We define the mean square displacement function $\psi(t)$ as the variance of $X_{\alpha}$ at $t$, given that the initial distribution of the monomers was $P_{\text {ni }}(\{\mathbf{a}\})$ given by (54). Because $\mathbf{X}=\sum b_{i} \mathbf{a}_{i}$, we have $\psi(t)=\sum_{i, j=1}^{N} b_{i} b_{j} \gamma_{i j}(t)$, and therefore:

$$
\psi(t) \equiv \frac{1}{d}\left\langle\left[\mathbf{X}(t)-\mathbf{X}_{0}\right]^{2}\right\rangle=2 D b_{1}^{2} t+2 D \sum_{j \geqslant 2} \frac{b_{j}^{2}}{\lambda_{j}}\left(1-\mathrm{e}^{-\lambda_{j} t}\right),
$$

where $d$ is the space dimension. Then, we can define an 'effective propagator' (or 'reduced Green function') in free space:

$$
P\left(\mathbf{X}, t \mid\left\{\mathbf{X}_{0}, \text { eq }\right\}, 0\right)=\frac{1}{[2 \pi \psi(t)]^{d / 2}} \exp \left\{-\frac{\left(\mathbf{X}-\mathbf{X}_{0}\right)^{2}}{2 \psi(t)}\right\}
$$

Note that this represents the probability of finding the reactive monomer at position $\mathbf{X}$ at time $t$, given that it was observed at position $\mathbf{X}_{0}$ at the initial time $t=0$, and that the rest of the chain was at equilibrium at this initial time. We stress that the effective propagator (59) does not satisfy the Chapman Kolmogorov equation: 
$P\left(\mathbf{X}, t \mid\left\{\mathbf{X}_{0}\right.\right.$, eq $\left.\}, 0\right) \neq \int \mathrm{d} \mathbf{X}^{\prime} P\left(\mathbf{X}, t \mid\left\{\mathbf{X}^{\prime}\right.\right.$, eq $\left.\}, t^{\prime}\right) P\left(\mathbf{X}^{\prime}, t^{\prime} \mid\left\{\mathbf{X}_{0}\right.\right.$, eq $\left.\}, 0\right)$,

reflecting the fact that the process $\mathbf{X}(t)$ is not Markovian. It is however tempting to use (59) as an effective propagator in the expression of the MFPT derived for Markovian processes (see (40)):

$$
\lim _{V \rightarrow \infty} \frac{\langle\mathbf{T}\rangle_{\text {Markovian }}}{V}=\int_{0}^{\infty} \frac{\mathrm{d} t}{[2 \pi \psi(t)]^{d / 2}}\left\{\exp \left(-\frac{a^{2}}{2 \psi(t)}\right)-\exp \left(-\frac{\left|\mathbf{X}_{0}\right|^{2}}{2 \psi(t)}\right)\right\} .
$$

We call this assumption the Markovian assumption, and we will see in section 4.1 that in the case of cyclization this assumption is equivalent to the Wilemski Fixman approximation. This formula suggests that as in the Markovian case, the MFPT is proportional to the volume, and that its dependance with the initial distance is contained in the infinite space effective propagator, which depends on $\psi(t)$. More precisely, in the case of the Rouse chain, it can be shown by calculating explicitly the eigenvalues $\lambda_{i}$ and coefficients $b_{i}$ that:

$$
\psi(t) \simeq \begin{cases}2 D t & \text { if } t \ll \tau_{0} \\ \kappa t^{1 / 2} & \text { if } \tau_{0} \ll t \ll N^{2} \tau_{0} \\ 2 D_{\mathrm{cm}} t & \text { if } t \gg \tau_{0} N^{2}\end{cases}
$$

where $D_{\mathrm{cm}}=D b_{1}^{2}=D / N$ is the diffusion coefficient of the center- of-mass, $\tau_{0}=\zeta / k$ is the relaxation time of a single bond, and $\kappa=4 l_{0}^{2} / \sqrt{\pi \tau_{0}}$ for an end-monomer, with $l_{0}=\sqrt{k_{B} T / k}$ the equilibrium bond length. Equation (62) states that, at very short times scales, a monomer behaves as if it were disconnected from the rest of the chain and freely diffused. At intermediate time scales, all the internal time scales contribute to the motion and it is known that the monomer motion becomes subdiffusive. Non-Markovian subdiffusion is characteristics of polymer dynamics, with exponents that depend on the model considered [85]. At larger time scales, the monomer again has a diffusive motion, due to the motion of the polymer center-of-mass.

Using the properties (62) of the function $\psi(t)$, we can infer those of the MFPT in the Markovian approximation using the formula (61) if $d=1$ (with a target size $a=0$ ) [91]:

$$
\langle\mathbf{T}\rangle_{\text {Markovian }} \simeq V \times \begin{cases}2 X_{0} / D & \text { if } X_{0} \ll l_{0} \\ 0.3516 X_{0}^{2} N^{1 / 2} / D l_{0} & \text { if } l_{0} \ll X_{0} \ll l_{0} N^{1 / 2} \\ 2 X_{0} / D_{\mathrm{cm}} & \text { if } X_{0} \gg l_{0} N^{1 / 2}\end{cases}
$$

Interestingly, in the intermediate scale regime, the MFPT is thus found to scale as $X_{0}^{2}$. This is in contradiction with the results $T \simeq X_{0}^{3}$ obtained from a naive application of equation (46) with a walk dimension $d_{w}=4$ and a space dimension $d=1$. In fact, we will see that in this regime non-Markovian corrections lead to the scaling $T \simeq X_{0}^{3}$ (see equation (75)).

In 3D, the Markovian approximation of the MFPT reads in the limit of large initial distance between the target and the polymer $\left(\left|X_{0}\right| \rightarrow \infty\right)$ :

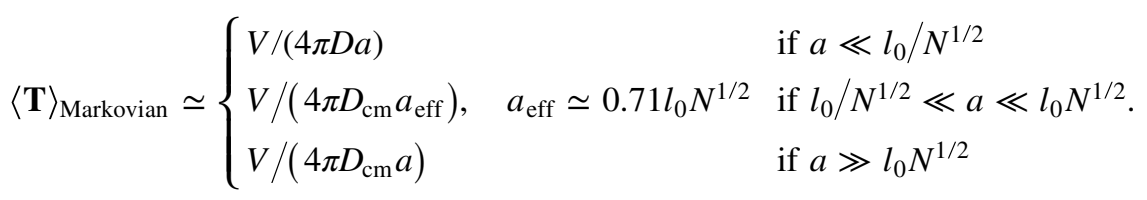

where $a_{\mathrm{eff}}$ is an effective reactive radius. Comparing with the expression for a Markovian diffusive walker (equation (36)), we observe that, at very small target sizes, the MFPT is the 
same as that of an isolated reactive monomer and is essentially controlled by the microscopic diffusion coefficient of the individual monomer. The most interesting regime is the intermediate regime, where the MFPT is that of an effective particle of diffusion coefficient $D_{\mathrm{cm}}$ that reacts with a target whose size $a_{\text {eff }}$ is larger than the 'true' target size $a$. This size $a$ does not enter in the expression of the MFPT reflecting the compact feature of the walk at length scales between $l_{0}$ and $l_{0} N^{1 / 2}$. Finally, for target sizes much larger than the gyration radius, the polymer behaves as a single particle of diffusion coefficient $D_{\mathrm{cm}}=D / N$.

\subsection{The Renewal equation: non-Markovian case}

We now adapt the Renewal equation method that was used in the case of Markovian processes (equations (2) (8)) to this non-Markovian problem, following the works of [91, 92]. We focus here on the 1D problem for simplicity; generalization to 3D will be described in the next section. The target is assumed to be located at position $X=0$. While the dynamics of the position of the reactive monomer $X(t)$ is non-Markovian, we can use the fact that the problem with $N$ degrees of freedom is Markovian to write the following Renewal equation, valid for any configuration $\{a\}$ such that $X=\sum_{i} b_{i} a_{i}=0$ :

$P(\{a\}, t \mid\{$ ini $\}, 0)=\int_{0}^{t} \mathrm{~d} t^{\prime} \int \mathrm{d}\left\{a^{\prime}\right\} f\left(\left\{a^{\prime}\right\}, t^{\prime}\right) P\left(\{a\}, t-t^{\prime} \mid\left\{a^{\prime}\right\}, 0\right)$.

Here, $f\left(\left\{a^{\prime}\right\}, t^{\prime}\right)$ is the probability density to reach the target for the first time at time $\mathrm{t}^{\prime}$, with a configuration $\left\{a^{\prime}\right\}=\left(a_{1}^{\prime}, \ldots, a_{N}^{\prime}\right)$. In turn, $P\left(\left\{a^{\prime}\right\}, t \mid\{a\}, 0\right)$ is the propagator of the chain in the presence of confinement, but in the absence of absorption at $X=0$, and $P\left(\left\{a^{\prime}\right\}, t \mid\{\right.$ ini $\left.\}, 0\right)$ is the probability to observe the configuration $\left\{a^{\prime}\right\}$ at $t$, starting from the initial distribution $P_{\text {ini }}(\{a\})$ (equation 54)). We introduce the splitting probability distribution $\pi(\{a\})$ that represents the probability density of observing a configuration $|a\rangle$ when the reaction takes place:

$$
\pi(\{a\}) \equiv \int_{0}^{\infty} \mathrm{d} t f(\{a\}, t) .
$$

Taking the Laplace transform of the renewal equation (65) and developing for small values of the Laplace variable (adapting the steps used in equations (5), (7), (8)), we obtain that the distribution $\pi$ is normalized, and that:

$$
\langle\mathbf{T}\rangle P_{\mathrm{eq}}(\{a\})=\int_{0}^{\infty} \mathrm{d} t[P(\{a\}, t \mid \pi, 0)-P(\{a\}, t \mid\{\text { ini }\}, 0)]
$$

which is valid for all the conformations $\{a\}$ such that $\sum_{i} b_{i} a_{i}=0$. Here we have introduced $P_{\text {eq }}(\{a\})$ that represents the probability of observing a given configuration in the equilibrium state in confinement. If the confining volume is sufficiently large, the stationary distribution is equal to:

$$
P_{\mathrm{eq}}(\{a\}) \simeq \frac{\left|b_{1}\right|}{V}\left[\frac{\lambda_{2} \ldots \lambda_{N}}{(2 \pi D)^{N-1}}\right]^{1 / 2} \exp \left[-\sum_{i=2}^{N} \frac{\lambda_{i} a_{i}^{2}}{2 D}\right],
$$

which means that the center-of-mass is uniformly distributed in the volume $V$, while all the other internal degrees of freedom of the chain are at equilibrium and do not feel the confining volume.

In (67), the quantity $P(\{a\}, t \mid \pi, 0)$ is the probability of a configuration $\{a\}$ at $t$ given that the configuration at $t=0$ is taken from the splitting probability $\pi$. It is given by the convolution equation: 


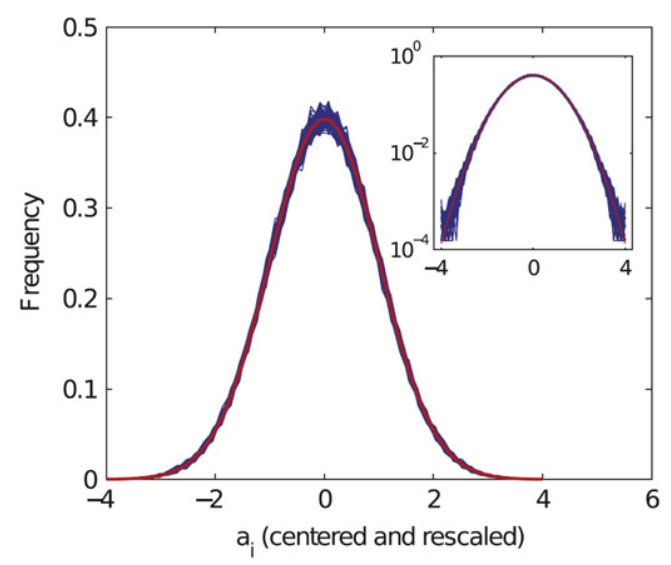

Figure 8. Histograms of mode positions $a_{i}$, after they have been centered and rescaled to have a zero mean and variance 1 , obtained from numerical (Langevin) simulations of the evolution of a Rouse chain of $N=20$ monomers, whose first monomer is seeking for a target located at $X=0$. The figure is a superposition of 200 histograms $\pi\left(a_{i} ; X_{0}\right)$, for all initial distances $X_{0}$ appearing in figure equation (9, and all values of $i$ $(1 \leqslant i \leqslant N)$. Red curve: normalized Gaussian distribution. Inset: same plot, in semi log scale.

$$
P(\{a\}, t \mid \pi, 0)=\int \mathrm{d}\left\{a^{\prime}\right\} \pi\left(\left\{a^{\prime}\right\}\right) P\left(\{a\}, t \mid\left\{a^{\prime}\right\}, 0\right) .
$$

The equations (67), (69) together with the normalization of $\pi$ form an integral equation that completely defines the splitting probability $\pi$ and the mean first reaction time $T$. Its use is however limited since it is required to solve for an unknown function of $N$ variables, with $N$ potentially large.

To go further, two additional steps can be followed:

(i) Large volume limit: in this limit, all the terms appearing in the right-hand side of equation (67) can be replaced by their expression in unbounded space, as previously done in the case of Markovian walkers, see equations (20), (44).

(ii) Gaussian approximation of the splitting distribution: we assume that the splitting probability distribution $\pi(\{a\})$ can be accurately described by a multivariate Gaussian distribution, with mean vector $m_{i}^{\pi}$ and covariance matrix $\sigma_{i j}^{\pi}$ that are determined by solving a set of self-consistent equations. This Gaussian approximation is well supported by numerical simulations (figure 8 ).

With these assumptions, the evolution of the average value $\mu_{i}^{\pi}(t)$ of the mode $a_{i}$ at a time $t$ after the first passage to the target satisfies $\partial_{t} \mu_{i}^{\pi}=-\lambda_{i} \mu_{i}^{\pi}$, leading to:

$$
\mu_{i}^{\pi}(t)=m_{i}^{\pi} \mathrm{e}^{-\lambda_{i} t}
$$

We next define $X_{\pi}(t)$ as the average of $X$ at a time $t$ after the first passage to the target. Because $X=\sum_{j} b_{j} a_{j}$, it is simply given by:

$$
X_{\pi}(t) \equiv \sum_{i=1}^{N} b_{i} \mu_{i}^{\pi}(t)=-\sum_{i=2}^{N} b_{i} m_{i}^{\pi}\left(1-\mathrm{e}^{-\lambda_{i} t}\right)
$$

where we have used the fact that $X_{\pi}(0)=0$. For simplicity of the presentation, we assume that the covariance matrix $\sigma_{i j}^{\pi}$ can be approximated by the covariance of equilibrium 
configurations constrained by $X=0: \sigma_{i j}^{\pi} \simeq \sigma_{i j}^{\text {eq,* }}$ given by equation (55). We call this additional approximation the 'stationary covariance approximation'. With these hypotheses, the distribution $P(\{a\}, t \mid \pi, 0)$ of configurations at a time $t$ after the first passage is also a Gaussian, with covariance matrix $\gamma_{i j}(t)$ (equation (57)), and mean vector $\mu_{i}^{\pi}(t)$.

The value of the modes at the instant of first passage, $m_{i}{ }^{\pi}$, will be deduced from a set of self-consistent equations. The derivation of these equations requires the calculation of the following general Gaussian integrals:

$$
\begin{aligned}
\int \mathrm{d}\{a\} a_{i} \delta\left(\sum_{j=1}^{N} b_{j} a_{j}\right) & \exp \left\{-\frac{1}{2} \sum_{j, k=1}^{N}\left(a_{j}-\mu_{j}\right)\left(\gamma^{-1}\right)_{j k}\left(a_{k}-\mu_{k}\right)\right\} \\
& =\left[\mu_{i}-\frac{\left\langle e_{i}|\gamma| b\right\rangle}{\langle b|\gamma| b\rangle}\langle b \mid \mu\rangle\right]\left[\frac{\operatorname{det}(2 \pi \gamma)}{2 \pi\langle b|\gamma| b\rangle}\right]^{1 / 2} \exp \left[-\frac{\langle b \mid \mu\rangle^{2}}{2\langle b|\gamma| b\rangle}\right],
\end{aligned}
$$

where we adopted the notation $\langle v|M| u\rangle=\sum_{i, j} v_{i} M_{i j} u_{j}$ for any symmetric matrix $M$ and vectors $\{u\},\{v\}$, and $\left|e_{i}\right\rangle$ is the vector whose elements are all zero, except for the $i$ th which takes the value 1 . Using the formula (72), the result of the multiplication of the integral equation (67) by $a_{i} \delta\left(\sum_{j} b_{j} a_{j}\right)$ gives the following set of equations, for any $i$ between 2 and $N$ :

$$
\begin{gathered}
0=\int_{0}^{\infty} \frac{\mathrm{d} t}{\psi(t)^{1 / 2}}\left\{\exp \left(-\frac{\left[X_{\pi}(t)\right]^{2}}{2 \psi(t)}\right)\left[m_{i}^{\pi} \mathrm{e}^{-\lambda_{\mathrm{i}} t}-\frac{b_{i}\left(1-\mathrm{e}^{-\lambda_{\mathrm{i}} t}\right) X_{\pi}(t)}{\lambda_{\mathrm{i}} \psi(t)}\right]\right. \\
\left.+\exp \left(-\frac{X_{0}^{2}}{2 \psi(t)}\right) \frac{b_{i}\left(1-\mathrm{e}^{-\lambda_{\mathrm{i}} t}\right) X_{0}}{\lambda_{\mathrm{i}} \psi(t)}\right\}
\end{gathered}
$$

The equations (73), together with the expression of $X_{\pi}(t)$ given by (71), form a set of $N$ selfconsistent non-linear equations that completely define the moments $m_{i}{ }^{\pi}$. An expression of the MFPT is obtained from (67), after multiplication by $\delta\left(\sum_{i} b_{i} a_{i}\right)$ and integration over all configurations:

$$
\frac{\langle\mathbf{T}\rangle}{V}=\int_{0}^{\infty} \frac{\mathrm{d} t}{[2 \pi \psi(t)]^{1 / 2}}\left\{\exp \left(-\frac{\left[X_{\pi}(t)\right]^{2}}{2 \psi(t)}\right)-\exp \left(-\frac{X_{0}^{2}}{2 \psi(t)}\right)\right\} .
$$

This expression finally gives the MFPT, and fully takes into account non-Markovian effects. Some remarks can be made at this stage:

(i) Structure of the equations and scaling with the volume. Comparing equation (74) with the expression of the MFPT obtained in the Markovian approximation (61), as well as with those obtained for Markovian walkers (20) and (44), we see that the MFPT is still proportional to the volume. A key ingredient of this approach is the quantity $X_{\pi}(t)$, which represents the fictive averaged trajectory followed by the walker in the future of the first passage event. This directly depends on the averaged configuration of the chain at the first passage event, described by the $m_{i}{ }^{\pi}$. Note that the Markovian approximation is recovered by setting $m_{i}^{\pi}=0$.

(ii) Validity of the non-Markovian theory. On figure 9, we compare the predictions of the MFPT for different theories to the results of simulations for the Rouse chain. If the Markovian approximation clearly overestimates the MFPT, the non-Markovian theory is 


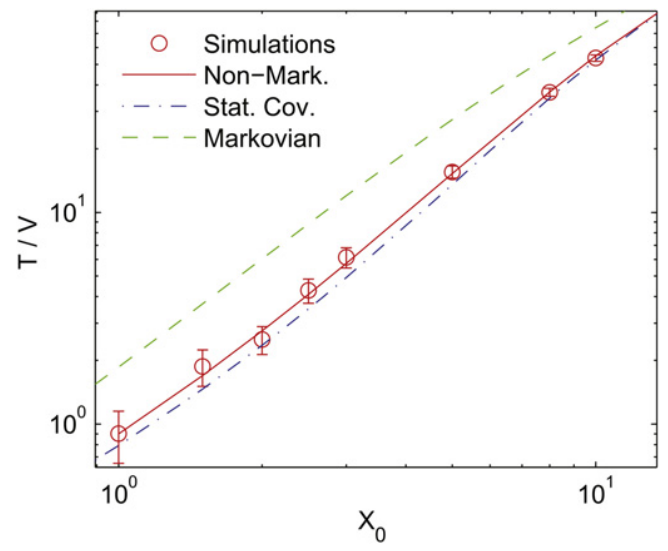

Figure 9. MFPT of the first monomer of a Rouse chain to reach a target located at $X=0$, as a function of initial distance $X_{0}$ for $N=20$ monomers. Symbols: stochastic simulations, dashed upper green line: Markovian approximation (61), lower dashed blue line: non Markovian prediction obtained by numerically solving (73), red full line: non Markovian theory without doing the stationary covariance approximation, described in [91]. The polymer evolves inside a confining volume of radius $R=40.25 l_{0}$. Lengths and times are in units of $l_{0}$ and $\tau_{0}$, respectively.

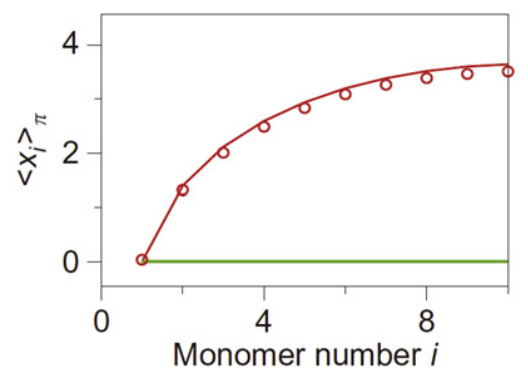

Figure 10. Average of monomer positions at the instant of the first passage of the first monomer to the target, for $N=10$ and $X_{0}$ large in 1D. Symbols: numerical (Langevin) simulations, upper red line: non Markovian theory, lower green line: Markovian theory. The unit length is $l_{0}$.

in quantitative agreement with the simulations. Note that the 'stationary covariance approximation' is accurate, as shown in figure 9.

(iii) Meaning of the non-Markovian theory. It is clear on figure 9 that the non-Markovian theory predicts MFPTs that are much lower than in the Markovian approximation. This can be understood by considering the average position of the monomers at the instant of the reaction, $\left\langle x_{i}\right\rangle_{\pi}=\sum_{j=1}^{N} Q_{j i} m_{j}^{\pi}$ (see (51)), represented in figure 10. It is seen on this plot that the positions of the non-reactive monomers of the chain are shifted on average at the instant of first passage. Therefore, the non-Markovian theory takes into account the events, due to fluctuations, which bring the first monomer to the target, while the rest of the monomers, and the polymer center-of-mass, are still far from the target. This leads to a smaller estimate of the MFPT than in the Markovian approximation, which does not 
account for these events correctly, since it assumes that the reactive conformations of the chain are equilibrium conformations.

(iv) Strongly non-Markovian regime. A careful analysis [91] of the asymptotic properties of equations (73), (74) reveals that:

$$
\langle\mathbf{T}\rangle \simeq V \times \begin{cases}2 X_{0} / D & \text { if } X_{0} \ll l_{0} \\ \tilde{\kappa} X_{0}^{3} & \text { if } l_{0} \ll X_{0} \ll l_{0} N^{1 / 2} \\ 2 N X_{0} / D & \text { if } l_{0} N^{1 / 2} \ll X_{0}\end{cases}
$$

with $\tilde{\kappa}$ a constant independent of $N$ and $X_{0}$. Comparing with (63), we see that the regime at intermediate length scales is different from that predicted by the Markovian approximation. Here one finds $T \sim V X_{0}^{d_{w}-d_{f}}$ with $d_{w}=4$ and $d_{f}=1$, as could be expected from a naive application of equation (46), originally derived for scale-invariant Markovian processes. In addition, the scalings for small $X_{0}$ and large $X_{0}$ are the same as predicted for Markovian processes (see equation (63)). At very short length scales, the process reduces to the free diffusion of an isolated reactive monomer, while at very large length scales it is controlled by the diffusion of the center-of-mass.

\subsection{Non-Markovian theory in $3 D$}

We now consider the extension of the theory to $3 \mathrm{D}$. The problem consists in determining the MFPT of a reactive monomer to a target of radius $a>0$. The main difference with the 1D case is that the extension $a$ of the target must now be taken into account. We denote by $\hat{\mathbf{u}}$ the 'arrival direction', defined as the unit vector normal to the target sphere at the position of the reactive monomer at its first passage to the target. Anticipating that, when the confinement volume is large, the MFPT will depend only on the initial distance between the polymer and the target, we assume isotropic initial conditions. Then, the arrival direction vector $\hat{\mathbf{u}}$ is isotropically distributed. The main hypothesis of the non-Markovian theory is that the distribution of conformations of the chain at the instant of first passage, given that the arrival direction is $\hat{\mathbf{u}}$, is a multivariate Gaussian, with an average vector $m_{i}{ }^{\pi} \hat{\mathbf{u}}$. In the stationary covariance approximation, we also assume that the covariance matrix is isotropic and given by equation (55). We demote by $X_{\pi}(t)$ the average position of the reactive monomer at a time $t$ after the first passage in the direction $\hat{\mathbf{u}}$ by:

$$
\mathbf{X}_{\pi}(t)=X_{\pi}(t) \hat{\mathbf{u}}=\left[a-\sum_{i=2}^{N} b_{i} m_{i}^{\pi}\left(1-\mathrm{e}^{-\lambda_{i} t}\right)\right] \hat{\mathbf{u}} .
$$

By construction, $X_{\pi}(0)=a$. Then, following the same steps as in 1D (see [91]), we obtain the set of non-linear self-consistent equations that define $m_{i}^{\pi}$ :

$$
\begin{aligned}
& \int_{0}^{\infty} \mathrm{d} t\left\{\left[\frac{X_{\pi}(t) m_{i}^{\pi} \mathrm{e}^{-\lambda_{i} t}}{3}+\left(1-\frac{\left[X_{\pi}(t)\right]^{2}}{3 \psi(t)}\right) \frac{b_{i}\left(1-\mathrm{e}^{-\lambda_{i} t}\right)}{\lambda_{i}}\right] \mathrm{e}^{-\left[X_{\pi}(t)\right]^{2} /[2 \psi(t)]}\right. \\
& \left.-\left(1-\frac{\left|\mathbf{X}_{0}\right|^{2}}{3 \psi}\right) \frac{b_{i}\left(1-\mathrm{e}^{-\lambda_{i} t}\right)}{\lambda_{i}} \mathrm{e}^{-\left|\mathbf{X}_{0}\right|^{2} /[2 \psi(t)]}\right\} \frac{1}{\psi(t)^{5 / 2}}=0 .
\end{aligned}
$$




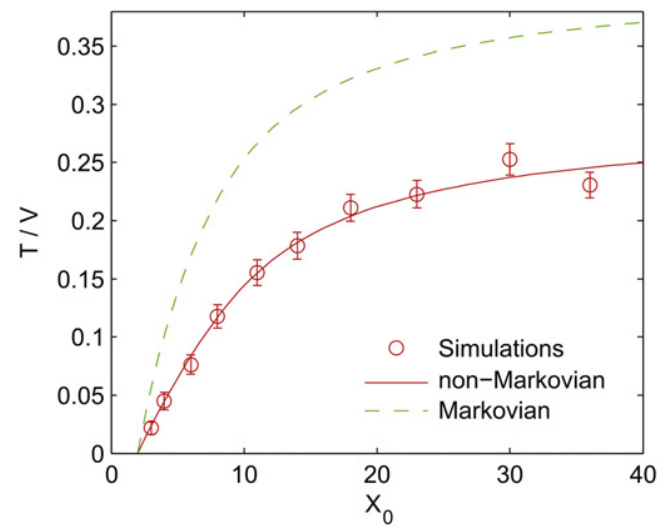

Figure 11. MFPT in 3D for a Rouse chain with $N=20$ monomers. The reactive monomer is the first monomer of the chain, the target is centered around 0 and its radius is $a=2 l_{0}$. Symbols: simulations, continuous red line: non Markovian theory (77), equation (78), upper dashed line: MFPT in the Markovian approximation (61).

Once the moments $m_{i}{ }^{\pi}$ are determined, the MFPT is calculated from:

$$
\frac{\langle\mathbf{T}\rangle}{V}=\int_{0}^{\infty} \frac{\mathrm{d} t}{[2 \pi \psi(t)]^{\frac{3}{2}}}\left[\exp \left(-\frac{\left[X_{\pi}(t)\right]^{2}}{2 \psi(t)}\right)-\exp \left(-\frac{\left|\mathbf{X}_{0}\right|^{2}}{2 \psi(t)}\right)\right] .
$$

Comparison with the expression (61) in the Markovian approximation shows that the key ingredient of the non-Markovian theory is to take into account the mean reactive trajectory $X_{\pi}(t)$ followed by the polymer after the reaction. The MFPT is represented as a function of the initial distance between the reactants in figure 11, where one observes that the use of the non-Markovian theory improves significantly the accuracy of the MFPT predictions.

A careful asymptotic analysis for large $V$ of the equations (76), (77), (78) shows that:

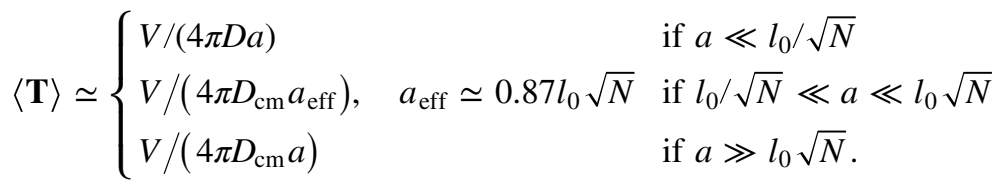

Comparing with the asymptotic expression (64) of the MFPT in the Markovian approximation, it appears that non-Markovian effects vanish in the regimes where the dynamics of the reactive monomer is purely diffusive (very large and very small target sizes in (79)). In the intermediate regime however, which involves a subdiffusive scaling, the nonMarkovian effects are quantitatively important, since the effective reaction radius $a_{\text {eff }}$ in (79) is significantly larger than in the Markovian theory. The configuration of the polymer at the instant of reaction is represented in figure 12. Similarly to the 1D case, the non-Markovian theory predicts that the chain is elongated in the direction of reaction, thereby taking into account the events where the first monomer hits the target due to fluctuations, while the center-of-mass is still far from it. 

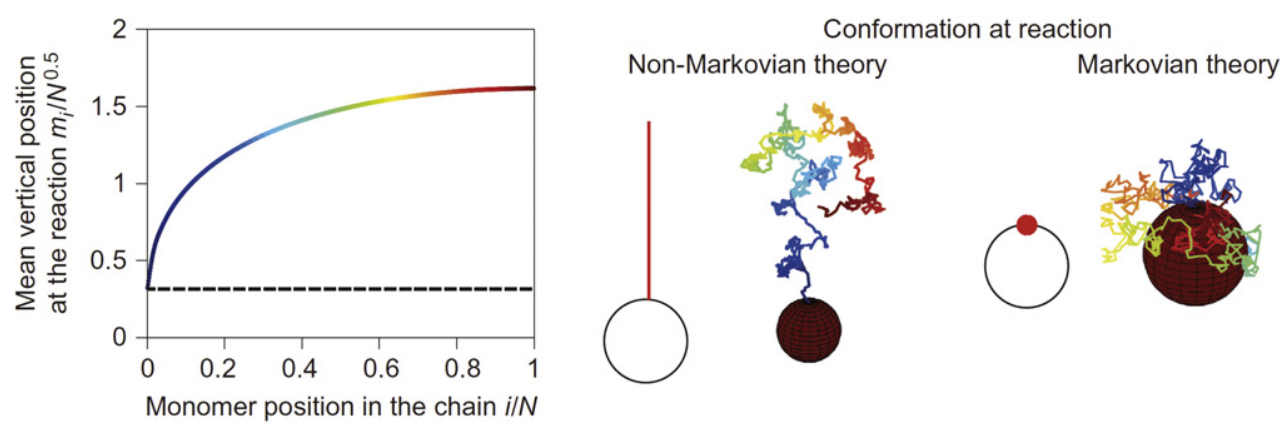

Figure 12. Predicted polymer conformations at reaction. Left: Average vertical position (the reaction is assumed to take place along the vertical axis, $\hat{\mathbf{u}}=\mathbf{e}_{z}$ ) of the monomers when the reaction takes place for the reaction between the first monomer and a target (continuous line: prediction of non Markovian theory, dashed line: Markovian approximation). We also plot the sketch of the polymer shape when the reaction takes place, and an example of conformation drawn from the splitting probability distribution (left), which is in marked contrast with the stationary distribution (right). The position of a monomer in the chain is represented by a colour code. Parameters: $N=800$ and $a=0.32 l_{0} N^{1 / 2}$

\section{Cyclization dynamics of polymer chains}

We now consider the cyclization dynamics of a Rouse chain. The determination of the mean time before two momomers of the same polymer chain meet is an old problem of statistical mechanics. It has led to intense theoretical efforts and we shall discuss here important theoretical approaches, including the early theories of Doi [93], Wilemski Fixman [94, 95], Szabo, Schulten and Schulten [96], and also recent theoretical advances [97 102], which we will summarize in this section. The cyclization time can be seen as an FTP in an effective confinement volume whose diameter is of the order of the chain gyration radius. Note that the end-to-end vector, denoted $\mathbf{X}_{\mathrm{ee}}(t)$, is a non-Markovian variable, which formally makes the cyclisation problem similar to the question analyzed in previous section.

We still consider a chain of monomers of positions $\mathbf{x}_{1}, \ldots, \mathbf{x}_{N}$, whose dynamics is given by the Fokker Planck equation (49). The dynamics can be equivalently defined by considering the modes $\{\mathbf{a}(t)\}$ defined by equation (51) and evolving independently with equation (52). The variable of interest is now the is the end-to-end distance,

$$
\mathbf{X}_{\mathrm{ee}}(t)=\mathbf{x}_{N}(t)-\mathbf{x}_{1}(t),
$$

which can also be considered as a combination of the Rouse modes:

$$
\mathbf{X}_{\mathrm{ee}}(t)=\sum_{i=2}^{N} b_{i} \mathbf{a}_{i}, \quad b_{i}=Q_{N i}-Q_{1 i}
$$

Interestingly, $\mathbf{X}_{\mathrm{ee}}$ has no component on the first mode, which is proportional to the position of the center-of-mass. The expression of the 'effective propagator' (59) is modified, and is given by:

$$
P\left(\mathbf{X}_{\mathrm{ee}}, t \mid\left\{\mathbf{X}_{\mathrm{ee}}^{0}, \mathrm{eq}\right\}, 0\right)=\frac{1}{[2 \pi \psi(t)]^{d / 2}} \mathrm{e}^{-\left[\mathbf{X}_{\mathrm{ee}}-\mathbf{X}_{\mathrm{ee}}^{0} \phi(t)\right]^{2} /[2 \psi(t)]}
$$


where $d$ is the space dimension. The effective propagator can be interpreted as follows: if one measures the end-to-end distance $\mathbf{X}_{\mathrm{ee}}^{0}$ for a polymer at equilibrium at $t=0$, and let the polymer evolve, the distribution of $\mathbf{X}_{\mathrm{ee}}$ at time $t$ is then given by equation (82). Here, the function $\phi(t)$, which characterizes the dynamics, is the correlation function:

$$
\phi(t)=\frac{\left\langle\mathbf{X}_{\mathrm{ee}}(t) \mathbf{X}_{\mathrm{ee}}(0)\right\rangle}{\left\langle\mathbf{X}_{\mathrm{ee}}(0)^{2}\right\rangle}=\sum_{i=2}^{N} \frac{D b_{i}^{2} \mathrm{e}^{-\lambda_{i} t}}{\lambda_{\mathrm{i}} L^{2}}
$$

where $L$ is the equilibrium mean square end-to-end distance, defined as:

$$
L^{2}=\frac{1}{d}\left\langle\mathbf{X}_{\mathrm{ee}}(0)^{2}\right\rangle=\sum_{i=2}^{N} \frac{D b_{i}^{2}}{\lambda_{i}}
$$

and the mean square displacement function $\psi(t)$ is related to $\phi(t)$ by the relation

$$
\psi(t)=L^{2}\left[1-\phi(t)^{2}\right] .
$$

Note that $L=l_{0}(N-1)^{1 / 2}$ in the case of the Rouse chain. This quantity will be useful in the next sections, where we review various approaches to the cyclization problem.

\subsection{The Wilemski-Fixman approach}

We first describe the Wilemski Fixman [94, 95] approach and underline its links with the non-Markovian approach presented in section 4.2 (see also [103]). Wilemski and Fixman considered the irreversible cyclization of a chain, in the presence of a 'sink' term $k\left(\mathbf{X}_{\mathrm{ee}}\right)$, defined as absorption probability per unit time, which is assumed to depend on the end-to-end distance. In the presence of the sink term, the Fokker Planck equation (49) becomes:

$$
\frac{\partial \Psi}{\partial t}=\sum_{i=2}^{N} \nabla_{i} \lambda_{i} \mathbf{a}_{i} \Psi+D \sum_{i=2}^{N} \nabla_{i}^{2} \Psi-k\left(\mathbf{X}_{\mathrm{ee}}\right) \Psi
$$

where $\nabla_{i}=\partial / \partial_{a i}$. Note that here we call $\Psi(\{\mathbf{a}\}, t)$ the survival probability that the polymer is in conformation $\{\mathbf{a}\}$ at time $t$, and has not yet reacted with the sink. Now, let us call $P\left(\{\mathbf{a}\}, t \mid\left\{\mathbf{a}^{0}\right\}\right)$ the probability to observe the configuration $\{\mathbf{a}\}$ at $t$ in the absence of sink, given that $\left\{\mathbf{a}^{0}\right\}$ was observed at $t=0$. The solutions of (86) can then be written:

$P_{\mathrm{eq}}(\{\mathbf{a}\})=\Psi(\{\mathbf{a}\}, t)+\int_{0}^{t} \mathrm{~d} t^{\prime} \int \mathrm{d}\left\{\mathbf{a}^{\prime}\right\} P\left(\{\mathbf{a}\}, t-t^{\prime} \mid\left\{\mathbf{a}^{\prime}\right\}\right) k\left(\mathbf{X}_{\mathrm{ee}}^{\prime}\right) \Psi\left(\{\mathbf{a}\}^{\prime}, t^{\prime}\right)$.

This equation has a clear meaning: the probability to observe a configuration $\{\mathbf{a}\}$ at $t$ without sink, is the sum of the probability of being at $\{\mathbf{a}\}$ at $t$ without having reacted before (term $\Psi(\{\mathbf{a}\}, t)$ ), plus the probability of reaching $\{\mathbf{a}\}$ from $\left\{\mathbf{a}^{\prime}\right\}$ during a time $t-t^{\prime}$, multiplied by the probability that the reaction occurred at $\mathrm{t}^{\prime}$ with configuration $\left\{\mathbf{a}^{\prime}\right\}$. The probability density $f(\{\mathbf{a}\}, t)$ to react at $t$ with a configuration $\{\mathbf{a}\}$ is given by:

$$
f(\{\mathbf{a}\}, t)=k\left(\mathbf{X}_{\mathrm{ee}}\right) \Psi(\{\mathbf{a}\}, t)
$$

so that:

$P_{\mathrm{eq}}(\{\mathbf{a}\})=\frac{f(\{\mathbf{a}\}, t)}{k\left(\mathbf{X}_{\mathrm{ee}}\right)}+\int_{0}^{t} \mathrm{~d} t^{\prime} \int \mathrm{d}\left\{\mathbf{a}^{\prime}\right\} P\left(\{\mathbf{a}\}, t-t^{\prime} \mid\left\{\mathbf{a}^{\prime}\right\}\right) f\left(\{\mathbf{a}\}^{\prime}, t^{\prime}\right)$

which is the generalization of the Renewal equation (65) for finite $k$. Taking the temporal Laplace transform of (89) and expanding for small values of the Laplace variable, we get: 


$$
\langle\mathbf{T}\rangle P_{\mathrm{eq}}(\{\mathbf{a}\})=\frac{\pi(\{\mathbf{a}\})}{k\left(\mathbf{X}_{\mathrm{ee}}\right)}+\int_{0}^{\infty} \mathrm{d} t\left[P(\{\mathbf{a}\}, t \mid \pi)-P_{\mathrm{eq}}(\{\mathbf{a}\})\right],
$$

where $\pi(\{\mathbf{a}\})=\int_{0}^{\infty} \mathrm{dt} f(t,\{\mathbf{a}\})$ is the splitting probability, that is the probability to react with a configuration $\{\mathbf{a}\}$. Equation (90) is the generalization of the equation (67) for finite $k$. In the Wilemski Fixman approximation (see equation (41) of the original work of Wilemski Fixman [94]), one writes:

$$
\Psi(\{\mathbf{a}\}, t) \simeq P_{\mathrm{eq}}(\{\mathbf{a}\}) g(t)
$$

meaning that the sink does not modify much the distribution of the chain configurations. One can use this approximation to get an estimate for the density of FPT, however here we focus only on the MFPT. The approximation (91) is equivalent to:

$$
\pi(\{\mathbf{a}\}) \simeq \frac{P_{\mathrm{eq}}(\{\mathbf{a}\}) k\left(\mathbf{X}_{\mathrm{ee}}\right)}{\kappa_{\mathrm{eq}}}
$$

where the normalization constant $\kappa_{\mathrm{eq}}$ is the probability of absorption per unit time of a chain at equilibrium:

$$
\kappa_{\mathrm{eq}}=\int \mathrm{d}\{\mathbf{a}\} P_{\mathrm{eq}}(\{\mathbf{a}\}) k\left(\mathbf{X}_{\mathrm{ee}}\right)=\int \mathrm{d} \mathbf{X}_{\mathrm{ee}} P_{\mathrm{eq}}\left(\mathbf{X}_{\mathrm{ee}}\right) k\left(\mathbf{X}_{\mathrm{ee}}\right) .
$$

Now, we need to introduce a closure approximation, that is to find one single equation from (90) that will enable the determination $\langle\mathbf{T}\rangle$ in a self consistent way. The closure approximation of Wilemski Fixman consists in multiplying equation (90) by $k\left(\mathbf{X}_{\mathrm{ee}}\right)$ and integrating over all conformations, which leads to:

$$
\langle\mathbf{T}\rangle_{\mathrm{WF}}=\frac{1}{\kappa_{\mathrm{eq}}}+\int_{0}^{\infty} \mathrm{d} t[C(t)-1]
$$

where $C(t)$, called the sink sink correlation function, can be expressed by using the effective propagator (59):

$C(t)=\frac{1}{\kappa_{\mathrm{eq}}^{2}} \int \mathrm{d} \mathbf{X}_{\mathrm{ee}} \int \mathrm{d} \mathbf{X}_{\mathrm{ee}}^{\prime} k\left(\mathbf{X}_{\mathrm{ee}}\right) k\left(\mathbf{X}_{\mathrm{ee}}^{\prime}\right) P\left(\mathbf{X}_{\mathrm{ee}}, t \mid\left\{\mathbf{X}_{\mathrm{ee}}^{\prime}, \mathrm{eq}\right\}, 0\right) P_{\mathrm{eq}}\left(\mathbf{X}_{\mathrm{ee}}^{\prime}\right)$.

Here, this sink sink correlation function is normalized so that $C(\infty)=1$. Note also that $C(t)$ does not depend on the magnitude of the function $k$, but it depends only on its shape. Hence, the absorption time $\langle\mathbf{T}\rangle_{\mathrm{WF}}$ in equation (94) is the sum of two terms, the second of which is independent of the strength of the sink term and is the reaction time in the diffusion controlled limit.

An alternative, slightly different closure approximation can be done by multiplying equation (90) by $\delta\left(\mathbf{X}_{\mathrm{ee}}\right)$ instead of $k\left(\mathbf{X}_{\mathrm{ee}}\right)$ and integrating over the configurations, which leads to:

$$
C(t)=\frac{1}{\kappa_{\mathrm{eq}} P_{\mathrm{eq}}(\mathbf{0})} \int \mathrm{d} \mathbf{X}_{\mathrm{ee}}{ }^{\prime} k\left(\mathbf{X}_{\mathrm{ee}}{ }^{\prime}\right) P\left(\mathbf{0}, t \mid\left\{\mathbf{X}_{\mathrm{ee}}{ }^{\prime}, \mathrm{eq}\right\}, 0\right) P_{\mathrm{eq}}\left(\mathbf{X}_{\mathrm{ee}}{ }^{\prime}\right) .
$$

Interestingly, Wilemski and Fixman, after obtaining their expression (94), considered a supplementary approximation consisting in taking one of the sink terms equal to a delta function in (95) leading to equation (96) (see below equation (7) in [95]).

Now, we consider the diffusion controlled limit, and we address specifically the problem of finding the MFPT for $\mathbf{X}_{\mathrm{ee}}$ to reach $a$, which corresponds to the following form of the reaction rate: 


$$
k\left(\mathbf{X}_{\mathrm{ee}}\right)=k_{0} \delta\left(\left|\mathbf{X}_{\mathrm{ee}}\right|-a\right), \quad k_{0} \rightarrow \infty .
$$

In this case, inserting (97) into (94), (96) yields:

$$
\langle\mathbf{T}\rangle_{\mathrm{WF}} \simeq P_{\mathrm{eq}}(\mathbf{0})^{-1} \int_{0}^{\infty} \mathrm{d} t\left\{P\left(\mathbf{0}, t \mid\left\{\left|\mathbf{X}_{\mathrm{ee}}\right|=a, \text { eq }\right\}, 0\right)-P_{\mathrm{eq}}(\mathbf{0})\right\} .
$$

Since one would obtain exactly this expression by using the 'effective propagator' (82) in the expression of the MFPT obtained for Markovian walkers (40), the Wilemski Fixman approximation can be seen as a 'Markovian' approximation. It is also the generalization of the corresponding expression in the case of the reaction with a fixed target, equation (61).

It is instructive to look at the asymptotic relations predicted by the Wilemski Fixman expression (98) in the case of the Rouse chain, for which the MSD function $\psi(t)$ behaves as:

$$
\psi(t) \simeq \begin{cases}4 D t & \left(t \ll \tau_{0}\right) \\ \kappa \sqrt{t} & \left(\tau_{0} \ll t \ll \tau_{0} N^{2}\right) . \\ L^{2} & \left(t \gg \tau_{0} N^{2}\right)\end{cases}
$$

As in the case of the motion of one monomer (see equation (62)), we recover the diffusive and subdiffusive behavior at very small and small timescales, respectively. An asymptotic study of the Wilemski Fixman expression leads to:

$$
\langle\mathbf{T}\rangle_{\mathrm{WF}} \simeq \begin{cases}\sqrt{\pi / 8}\left[l_{0}(N-1)\right]^{3 / 2} /(D a) & \left(a \ll l_{0} / \sqrt{N}\right) \\ 0.201 N^{2} \tau_{0} & \left(l_{0} / N^{1 / 2} \ll a \ll l_{0} \sqrt{N}\right) .\end{cases}
$$

The first regime, for which $\langle\mathbf{T}\rangle \sim a^{-1}$ is simply the formula for a diffusive walker (equation (36)), with diffusion coefficient $2 D$ moving in an effective volume $V=P_{\mathrm{eq}}(\mathbf{0})^{-1}=\left(2 \pi L^{2}\right)^{3 / 2}$. In this regime, the monomers essentially behave as if they were diffusive molecules disconnected from the rest of the chain; no memory effects are thus expected, and the same asymptotic relation holds for the Wilemski Fixman and the nonMarkovian theories. For larger values of $N$, the cyclization time scales as $N^{2}$, and is independent of the capture radius, as expected for subdiffusive walks. The presence of two distinct regimes predicted by the Wilemski Fixman theory has been recognized by Pastor [104] and demonstrated by numerical stochastic simulations [105].

\subsection{Non-Markovian approach}

We describe in this section the adaptation of the theory presented in Sections (3.2), (3.3) to the problem of cyclization, as described in $[92,106]$. We remind that the problem is to calculate the MFPT of $\mathbf{X}_{\mathrm{ee}}$ to reach a reactive region of radius $a$, while the chain is assumed to be initially at equilibrium. Here, we assume in addition that initially the end-to-end distance is larger than $a$. Let us first notice that the Markovian approximation of equation (98) has to be adapted to take into account this supplementary constraint, and becomes [106]:

$$
\frac{\langle\mathbf{T}\rangle}{L^{3}}=\int_{0}^{\infty} \frac{\mathrm{d} t}{\psi(t)^{3 / 2}}\left[\exp \left(-\frac{[a \phi(t)]^{2}}{2 \psi(t)}\right)-\frac{Z(a, \psi(t))}{Z\left(a, L^{2}\right)}\right] .
$$


Here, the function $Z$ is defined as:

$$
Z(a, h)=\int_{a}^{\infty} \mathrm{d} R_{0} R_{0}^{2} \mathrm{e}^{-R_{0}^{2} /(2 h)}
$$

Equation (101) is obtained by doing a Markovian approximation, as in the Wilemski Fixman approximation.

Next, we show how to take into account non-Markovian effects. The starting point is the $3 \mathrm{D}$ version of the Renewal equation (67):

$$
\langle\mathbf{T}\rangle P_{\mathrm{eq}}(\{\mathbf{a}\})=\int_{0}^{\infty} \mathrm{d} t[P(\{\mathbf{a}\}, t \mid \pi, 0)-P(\{\mathbf{a}\}, t \mid\{\text { ini }\}, 0)]
$$

which is valid for any configuration $\{\mathbf{a}\}$ such that the end-to-end distance vector is inside the reactive region, $\left|\mathbf{X}_{\text {ee }}^{0}\right|=\left|\sum b_{i} \mathbf{a}_{i}\right| \leqslant a$. Multiplying equation (103) by $\delta\left(\sum b_{i} \mathbf{a}_{i}-\mathbf{X}_{\mathrm{ee}}\right)$ and integrating, we get:

$$
\langle\mathbf{T}\rangle P_{\mathrm{eq}}\left(\mathbf{X}_{\mathrm{ee}}\right)=\int_{0}^{\infty} \mathrm{d} t\left[P\left(\mathbf{X}_{\mathrm{ee}}, t \mid \pi, 0\right)-P\left(\mathbf{X}_{\mathrm{ee}}, t \mid\{\text { ini }\}, 0\right)\right],
$$

which is an exact expression of the MFPT, valid as soon as $\left|\mathbf{X}_{\text {ee }}\right| \leqslant a$. Comparing with equation (78), we observe that the stationary probability density of the end-to-end vector in the reactive region, $P_{\mathrm{eq}}\left(\mathbf{X}_{\mathrm{ee}}\right)$, plays the same role as the inverse of the volume for the problem with a fixed target. Note that the Markovian approximation (101) is recovered from (104) by assuming that the splitting distribution $\pi(\{\mathbf{a}\})$ is the equilibrium distribution, with the end-toend distance constrained to the surface of the sphere.

As in the 3D case for intermolecular reactions, we define the unit vector $\hat{\mathbf{u}}$ as the direction taken by $\mathbf{X}_{\mathrm{ee}}$ at the instant of first passage to the target, and we consider the distribution of reactive conformations $\pi_{\hat{\mathbf{u}}}(\{\mathbf{a}\})$, with the constraint that $\mathbf{X}_{\mathrm{ee}}=a \hat{\mathbf{u}}$. Then, in the Gaussian approximation, we assume that $\pi_{\hat{\mathbf{u}}}(\{\mathbf{a}\})$ is a multivariate Gaussian distribution, with mean vector $\mathbf{m}_{i}^{\pi}=m_{i}^{\pi} \hat{\mathbf{u}}$. For the sake of simplicity, we assume that the covariance matrix is approximated by the equilibrium covariance matrix, where $\mathbf{X}_{\mathrm{ee}}$ is constrained, which is given by the conditional covariance matrix [106]:

$$
\operatorname{cov}\left(a_{i, \alpha}, a_{j, \beta}\right)=\left(\frac{\delta_{i j}}{\lambda_{i}}-\frac{b_{i} b_{j}}{\lambda_{i} \lambda_{j} L^{2}}\right) \delta_{\alpha \beta} .
$$

We define the average trajectory $X_{\pi}(t)$ as the average value of $\mathbf{X}_{\mathrm{ee}}$. $\hat{\mathbf{u}}$ at a time $t$ after the firstpassage time, given that the direction of the end-to-end vector at the first passage was $\hat{\mathbf{u}}$ :

$$
X_{\pi}(t)=a-\sum_{i=2}^{N} b_{i} m_{i}^{\pi}\left(1-\mathrm{e}^{-\lambda_{i} t}\right) .
$$

The values of the moments $m_{i}^{\pi}$ are determined by a set of $N-1$ self-consistent equations, obtained by multiplying (103) by $a_{i} \delta\left(\mathbf{X}_{\mathrm{ee}}-\sum b_{j} \mathbf{a}_{j}\right)$, and by integrating over all conformations and then taking the limit $\left|\mathbf{X}_{\mathrm{ee}}\right| \rightarrow 0$ [106]: 


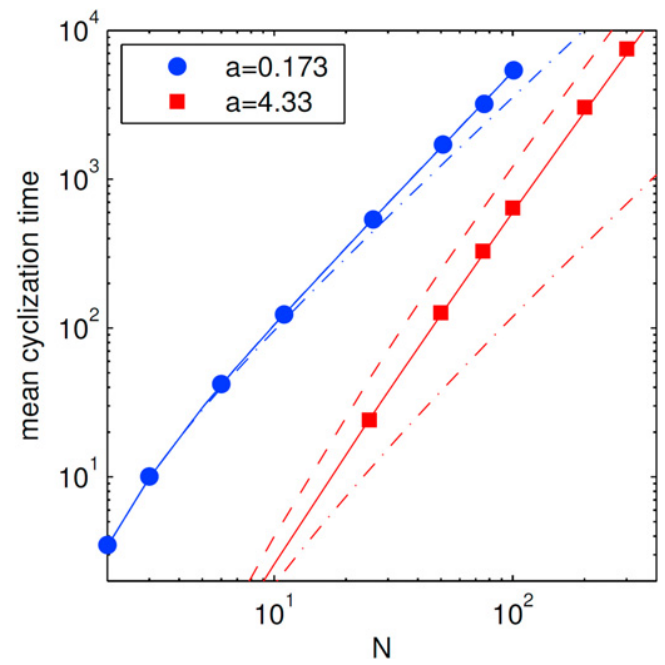

Figure 13. Average end to end cyclization time of a Rouse chain of $N$ monomers in simulations (symbols) and different theories (lines). There are two sets of curves, corresponding to two values of $a$, for which we represent the result of the non Markovian theory (full line), the Markovian (Wilemski Fixman) approximation (upper dashed lines), and the SSS approximation (lowed dashed dot line). For the smallest value of $a$, the non Markovian and the Markovian results are undistinguishable. The unit of time is the relaxation time of individual bonds, $\tau_{0}$.

$$
\begin{aligned}
\int_{0}^{\infty} \frac{\mathrm{d} t}{\psi^{5 / 2}}\left\{\left[\frac{m_{i}^{\pi} \mathrm{e}^{-\lambda_{i} t} X_{\pi}(t)}{3}+\frac{b_{i} \phi(t)\left[\phi(t)-\mathrm{e}^{-\lambda_{i} t}\right]}{\lambda_{i}}\left(1-\frac{X_{\pi}(t)^{2}}{3 \psi(t)}\right)\right] \mathrm{e}^{-\frac{\left.X_{Z}(t)\right)^{2}}{2 \psi(t)}}\right. \\
\left.-\frac{b_{i} \phi(t)\left[\phi(t)-\mathrm{e}^{-\lambda_{i} t}\right]}{Z\left(a, L^{2}\right) \lambda_{i}}\left[Z(a, \psi)-\frac{G(a, \psi(t))}{3 \psi(t)}\right]\right\}=0,
\end{aligned}
$$

where the function $G$ is defined by:

$$
G(a, h)=\int_{a}^{\infty} \mathrm{d} R_{0} R_{0}^{4} \mathrm{e}^{-R_{0}^{2} / 2 h} .
$$

The expression of the MFPT (104) finally becomes:

$$
\frac{\langle\mathbf{T}\rangle}{L^{3}}=\int_{0}^{\infty} \frac{\mathrm{d} t}{\psi(t)^{3 / 2}}\left[\exp \left(-\frac{X_{\pi}(t)^{2}}{2 \psi(t)}\right)-\frac{Z(a, \psi(t))}{Z\left(a, L^{2}\right)}\right] .
$$

Comparing with the Markovian approximation (101) (in which $X_{\pi}(t)=a \phi(t)$ ), we observe that, as in the case of a fixed target, the essential new feature of the non-Markovian theory is the presence of the average trajectory in the future of the first-passage time, $X_{\pi}(t)$, which is readily deduced from the reactive shape of the polymer through equation (106).

Examples of comparison between the predictions for the MFPT and simulations are presented in figure 13 for the Rouse chain, along with predictions of the SSS theory, in which the problem is mapped on the motion of an effective single particle diffusive in a harmonic potential (see section 4.3). We observe in figure 13 that, for small capture radius $a$, all theories predict with reasonable accuracy the MFPT, whereas for larger $a$ differences appear. An 

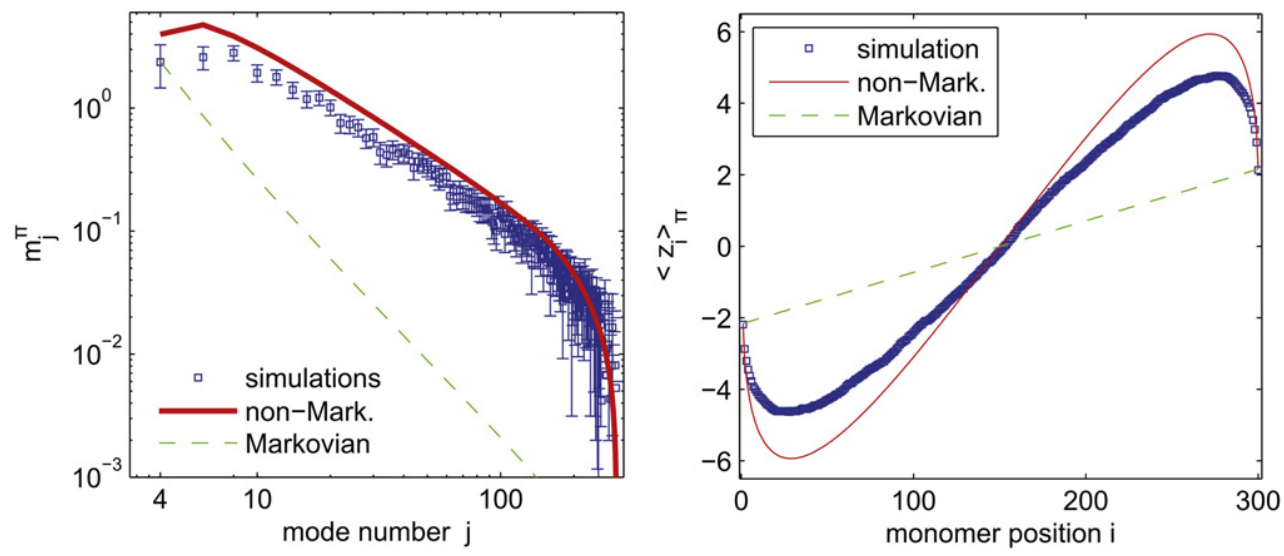

Figure 14. Left: Average value of the modes $m_{q}^{\pi}$ at the instant of end to end cyclization for a chain of $N=300$ monomers and a capture radius $a=4.33 l_{0}$. Symbols: simulations. Red line: non Markovian theory. Green thick dashed line: asymptotic relation of the non Markovian theory (110), proportional to $j^{4 / 3}$. Magenta thin dashed line: Markovian approximation. Right: average value of the monomer positions (in the direction of reaction, $\hat{\mathbf{u}})$ for the same parameters.

analysis of equations (106), (107), (109) reveals that the asymptotic scalings of the MFPT with $N$ and $a$ of the non-Markovian theory in 3D are the same as predicted by the Wilemski Fixman theory (see equation (100)). For small capture radius, all theories predict exactly the same asymptotic relation, $\langle\mathbf{T}\rangle \sim N^{3 / 2} / a$, with the same coefficient. We will see below that the result in this regime is in fact exact. However, in the regime $\langle\mathbf{T}\rangle \sim N^{2}$, the numerical prefactor is quantitatively different: the MFPT is in this regime controlled by the subdiffusive, nonMarkovian dynamics of the polymer at intermediate time scales.

In figure 14 , we represent an example of spectrum $m_{i}{ }^{\pi}$, obtained both from the selfconsistent equations (107) and numerical simulations. The agreement is very good, suggesting that the theory catches the essential characteristics of the spectrum of reactive conformations. Asymptotic analysis of equation (107) shows that, for large $q$, the spectrum has non-trivial power-law dependence with $q$ :

$$
m_{q}^{\pi} \simeq-\frac{2^{1 / 6} a^{1 / 3} N^{5 / 6}}{\pi q^{4 / 3}}
$$

which shows that the average positions of the monomers, $\langle\mathbf{x}(s)\rangle_{\pi}$ vary very fast next to the reactive monomers.

\subsection{The 'SSS' approach}

Another approach to compute the cyclization time is to approximate the non-Markovian dynamics of $\mathbf{X}_{\mathrm{ee}}$ by a simpler, Markovian dynamics, of an effective particle, of position $\mathbf{r}(t)$ moving in an effective potential $U(\mathbf{r})$, with an effective diffusion coefficient $D(\mathbf{r})$ for which the exact analytical calculation of the MFPT is possible. Following this strategy, a simple expression for the MFPT was presented in the theory developed by Szabo, Schulten and Schulten (SSS) [96], who proposed an alternative form to the earlier expressions of Sunagawa and Doi $[107,108]$. We will see however that the identification of the effective potential and effective diffusion coefficient is difficult. 
The Fokker Planck equation for the simplified dynamics is:

$$
\partial_{t} p(\mathbf{r}, t)=\beta \nabla[D(\mathbf{r})(\nabla U) p]+\nabla[D(\mathbf{r}) \nabla p]
$$

with $\beta=1 / k_{B} T$. The mean FPT $\tau(\mathbf{r})$ of a particle to a sphere of radius $a$ centered around $\mathbf{0}$, starting from position $\mathbf{r}$, obeys the adjoint, backward equation [96, 109]:

$$
-\beta D(\mathbf{r})(\nabla U) \nabla \tau(\mathbf{r})+\nabla[D(\mathbf{r}) \nabla \tau(\mathbf{r})]=-1 .
$$

The boundary condition at the surface of the target is $\tau(\mathbf{r})=0$ for $|\mathbf{r}|=a$. In the case where both the potential $U$ and the diffusion coefficient $D$ depend only on the radial coordinate $r=|\mathbf{r}|$, equation (112) is a first order differential equation for $\partial_{r} \tau(r)$ that can be solved explicitly. Averaging the solution over equilibrium initial configurations leads to [96]:

$$
\begin{aligned}
\langle\mathbf{T}\rangle_{\mathrm{SSS}} & =\frac{1}{\int_{a}^{\infty} \mathrm{d} r r^{2} \mathrm{e}^{-\beta U(r)}} \int_{a}^{\infty} \mathrm{d} r r^{2} \tau(r) \mathrm{e}^{-\beta U(r)} \\
& =\frac{1}{\int_{a}^{\infty} \mathrm{d} r r^{2} \mathrm{e}^{-\beta U(r)}} \int_{a}^{\infty} \frac{\mathrm{d} x}{D(x) x^{2} \mathrm{e}^{-\beta U(x)}}\left[\int_{x}^{\infty} \mathrm{d} y y^{2} \mathrm{e}^{-\beta U(y)}\right]^{2} .
\end{aligned}
$$

In the context of the cyclization of a Rouse chain, the potential $U(\mathbf{r})$ is identified with the harmonic potential whose stiffness is that of a spring formed by $N-1$ bonds in series: $U(\mathbf{r})=k_{\mathrm{eff}} \mathbf{r}^{2} / 2$ with the effective stiffness $k_{\mathrm{eff}}=k /(N-1)$. Assuming a constant diffusion coefficient for the effective particle modelling the end-to-end vector, and denoting $\tau_{\text {eff }}=k_{B} T /\left(D_{\text {eff }} k_{\text {eff }}\right)$ the effective relaxation time, equation (113) can be rewritten, in the limit $a \ll \sqrt{N}$ :

$$
\langle\mathbf{T}\rangle_{\mathrm{SSS}} \simeq \tau_{\mathrm{eff}} \frac{\sqrt{\pi}(N-1)^{1 / 2} l_{0}}{\sqrt{2} a} .
$$

Now, the choice of the effective diffusion coefficient is not straightforward. Pastor et al [104] have suggested that the optimal choice is $D_{\text {eff }}=2 D_{0}$, for which equation (114) coincides with the small $a$ limit of equation (100), $T \sim N^{3 / 2} / a$, with the same prefactor. However, the regime $T \sim N^{2}$, observed numerically, is not predicted by this approach. The main limitation of this approach is that it involves a single time scale, and therefore cannot reproduce all the observed regimes. In fact, as shown in figure 13, predictions of the SSS theory are not reliable.

Toan et al [98] have recently noticed that both $N^{2}$ and $N^{3 / 2} / a$ behaviors of (100) could however be recovered if one assumes that the diffusion coefficient depends on the length scale

$$
D_{\text {eff }}(r) \sim \begin{cases}D_{0} / \sqrt{N} & \left(r>l_{0}\right) \\ 2 D_{0} & \left(r<l_{0}\right) .\end{cases}
$$

Therefore, the work of [98] shows that the 'SSS' and the Wilemski Fixman results can be reconciliated, at the price of indroducing a space-dependent diffusion coefficient.

\subsection{Variational principles: upper and lower bounds on the MFPT}

Variational principles can be used to derive rigorous upper and lower bounds on the MFPT. Here, we follow the recent approach of Portman et al [101, 102], who generalized the early results of Doi [110]. The starting point is to consider the functional $M[\phi]$, defined for any 'trial' function $\phi(\{\mathbf{a}\})$ as (see equation (24) in [102]): 


$$
\begin{aligned}
M[\phi]= & \int \mathrm{d}\{\mathbf{a}\} k\left(\mathbf{X}_{\mathrm{ee}}\right) P_{\mathrm{eq}}(\{\mathbf{a}\})\left[\phi(\{\mathbf{a}\})^{2}-2 \phi(\{\mathbf{a}\})\right] \\
& +\int \mathrm{d}\{\mathbf{a}\} \int \mathrm{d}\left\{\mathbf{a}^{\prime}\right\} k(\{\mathbf{a}\}) \phi(\{\mathbf{a}\}) \hat{P}\left(\{\mathbf{a}\} s \mid\left\{\mathbf{a}^{\prime}\right\}\right) \\
& \times P_{\mathrm{eq}}\left(\left\{\mathbf{a}^{\prime}\right\}\right) k\left(\left\{\mathbf{a}^{\prime}\right\}\right) \phi\left(\left\{\mathbf{a}^{\prime}\right\}\right),
\end{aligned}
$$

where $\hat{P}\left(\{\mathbf{a}\}, s \mid\left\{\mathbf{a}^{\prime}\right\}\right)=\int_{0}^{\infty} \mathrm{d} t \mathrm{e}^{-s t} P\left(\{\mathbf{a}\}, t \mid\left\{\mathbf{a}^{\prime}\right\}\right)$ denotes the Laplace transform of the propagator without sink. The following remarks can be successively made:

(i) The quadratic term in $\phi$ of the functional (116) is positive, so that, for any function $\phi$, $M[\phi] \geqslant M\left[\phi^{*}\right]$, where $\phi^{*}$ satisfies $\delta M / \delta \phi^{*}=0$.

(ii) Calculating the functional derivative of (116) and comparing the result with equation (87), one obtains that the optimal function is $\phi^{*}(\{\mathbf{a}\})=\hat{\Psi}(\{\mathbf{a}\}, s) / P_{\mathrm{eq}}(\{\mathbf{a}\})$.

(iii) Evaluating $M$ at $\phi^{*}$ leads to $M\left[\phi^{*}\right]=-\hat{F}(s)=-1+\langle\mathbf{T}\rangle s+\mathcal{O}\left(s^{2}\right)$, where $\hat{F}(s)$ is the Laplace transform of the density of reaction time,

(iv) Evaluating $M$ for the constant trial function $\phi(\{\mathbf{a}\})=\bar{\phi}=\kappa_{\mathrm{eq}} /\left[\kappa_{\mathrm{eq}}+\hat{C}(s)\right]$, one finds that $M[\bar{\phi}] \simeq-1+s\langle\mathbf{T}\rangle_{\mathrm{WF}}$, which by virtue of the fact that $M$ is minimal at $\phi^{*}$, implies that:

$$
\langle\mathbf{T}\rangle \leqslant\langle\mathbf{T}\rangle_{\mathrm{WF}}
$$

which proves that the Wilemski Fixman approach gives an overestimate of the mean reaction time.

(v) A similar variational argument [101] can be used to show that the expression of the MFPT in the SSS approximation, with an effective diffusion coefficient $D_{\text {eff }}=2 D$ is a lower bound for the MFPT:

$$
\langle\mathbf{T}\rangle \geqslant\langle\mathbf{T}\rangle_{\mathrm{SSS}}
$$

(vi) Finally, since we have the two inequalities (117) and (118), and since both Wilemski Fixman and SSS approaches give the same asymptotic result in the limit $a \rightarrow 0$, we can deduce that the asymptotics $\langle\mathbf{T}\rangle \simeq N^{3 / 2} / a$ in equation (100) is exact in the limit $a \rightarrow 0$.

Other bounds can be found with this variational approach, if one uses different trial functions to evaluate the functional (116), the only limitation being the ability to perform the resulting integrals $[101,102]$.

\subsection{Strong localized perturbation theory}

Recently, Amitai et al [99] have proposed another approach of the cyclization problem, based on an expansion of eigenvalues of the Fokker Planck equation in the limit of small reactive radius, $a \rightarrow 0$. We present here a simplified version using the concepts of strong localized perturbation theory, as presented by Ward and Keller [111]. In the absence of absorbing region, the equilibrium distribution $P_{\mathrm{eq}}(\{\mathbf{a}\})$ is an eigenfunction of the Fokker Planck operator, with vanishing eigenvalue. When introducing a small reactive region of radius $a \rightarrow 0$, the vanishing eigenvalue $(\mu)$ is perturbed, as well as the associated eigenfunction (denoted $f(\{\mathbf{a}\}))$, that satisfy: 


$$
\begin{aligned}
\mu f(\{\mathbf{a}\}) & =\sum_{i=1}^{N} \lambda_{i} \nabla_{i}\left(\mathbf{a}_{i} f\right)+D \nabla_{i}^{2} f \\
& =D \sum_{i=1}^{N} \nabla_{i} P_{\mathrm{eq}}(\{\mathbf{a}\}) \nabla_{i} \frac{f(\{\mathbf{a}\})}{P_{\mathrm{eq}}(\{\mathbf{a}\})} .
\end{aligned}
$$

For $a \rightarrow 0$, the eigenfunction $f$ converges to $P_{\text {eq }}$, suggesting the expansion:

$$
f(\{\mathbf{a}\})=P_{\mathrm{eq}}(\{\mathbf{a}\})+a f_{1}(\{\mathbf{a}\})+\ldots \quad\left(\left|\mathbf{X}_{\mathrm{ee}}\right| \gg a\right) .
$$

However, this expansion cannot be valid close to the absorbing region, where $f$ must satisfy the absorbing boundary condition $f=0$ for $\left|\sum b_{i} \mathbf{a}_{i}\right|=a$. As a consequence, we assume that when $\left|\mathbf{X}_{\mathrm{ee}}\right| \sim a$, the function $f$ has a rapidly varying component $g$ and can be written:

$$
f(\{\mathbf{a}\}) \simeq h(\{\mathbf{a}\}) g\left(\mathbf{X}_{\mathrm{ee}} / a\right), \quad\left(\left|\mathbf{X}_{\mathrm{ee}}\right| \ll L\right)
$$

where $h$ and $g$ are functions independent of $a$. Inserting this last equation into the definition (119), and keeping only the leading order terms in $a$, we get:

$$
\nabla^{2} g=0 .
$$

Taking into account the absorbing boundary conditions at $\left|\mathbf{X}_{\mathrm{ee}}\right|=a$, the appropriate solution for $g$ is:

$$
g\left(\mathbf{X}_{\mathrm{ee}} / a\right)=C\left(1-a /\left|\mathbf{X}_{\mathrm{ee}}\right|\right) .
$$

Matching of the outer expansion (120) and the inner expansion (121), we find successively that $C h=P_{\text {eq }}$, and then that

$$
f_{1}(\{\mathbf{a}\}) \simeq-P_{\mathrm{eq}}(\{\mathbf{a}\}) /\left|\mathbf{X}_{\mathrm{ee}}\right| a \ll\left|\mathbf{X}_{\mathrm{ee}}\right| \ll L .
$$

Following closely the approach of [111], we introduce a small parameter $\sigma$, such that $a \ll \sigma \ll L$, and we integrate equation (119) over all configurations such that $\left|\mathbf{X}_{\mathrm{ee}}\right| \geqslant \sigma$, obtaining the following expression for the first eigenvalue in the limit of small $a$ :

$$
\mu \simeq D a \lim _{\sigma \rightarrow 0} \int_{\left|\mathbf{X}_{\mathrm{ee}}\right| \geqslant \sigma} \mathrm{d}\{\mathbf{a}\} \sum_{i=1}^{N} \nabla_{i} P_{\mathrm{eq}}(\{\mathbf{a}\}) \nabla_{i} \frac{f_{1}(\{\mathbf{a}\})}{P_{\mathrm{eq}}(\{\mathbf{a}\})} .
$$

The result of this integral would be zero if $f_{1}$ was a regular function. Replacing $f_{1}$ by its irregular behavior (124), we obtain:

$$
\mu=a D \lim _{\sigma \rightarrow 0} \int_{\left|\mathbf{X}_{\mathrm{ee}}\right| \geqslant \sigma} \mathrm{d}\{\mathbf{a}\} \sum_{i=1}^{N} \nabla_{i} P_{\mathrm{eq}}(\{\mathbf{a}\}) \nabla_{i} \frac{1}{\left|\mathbf{X}_{\mathrm{ee}}\right|} .
$$

The integration over the configurations leads to:

$$
\mu=a D\left(\sum_{i=1}^{N} b_{i}^{2}\right) \lim _{\sigma \rightarrow 0} \int_{\left|\mathbf{X}_{\mathrm{ee}}\right| \geqslant \sigma} \mathrm{d} \mathbf{X}_{\mathrm{ee}} \nabla_{\mathrm{ee}} P_{\mathrm{eq}}\left(\mathbf{X}_{\mathrm{ee}}\right) \nabla_{\mathrm{ee}} \frac{1}{\left|\mathbf{X}_{\mathrm{ee}}\right|} .
$$

The fact that $b_{i}=Q_{N i}-Q_{1 i}$ with $Q$ an orthogonal matrix implies that $\sum b_{i}^{2}=2$, and therefore the value of the perturbed eigenvalue is:

$$
\mu=8 \pi a D P_{\mathrm{eq}}(\mathbf{0}) .
$$

This eigenvalue is much lower than all other eigenvalues of the Fokker Planck equation in the limit $a \rightarrow 0$, and it therefore dominates the density of FPTs, which is approximately exponential in this regime, with an MFPT that reads: 


$$
\langle\mathbf{T}\rangle \simeq \mu^{-1}=\frac{\left[2 \pi l_{0}(N-1)\right]^{3 / 2}}{8 \pi a D} .
$$

Therefore, the result of equation (129) is exactly the one obtained by the Wilemski Fixman theory in the limit $a \rightarrow 0$, equation (100). Equation (129) is asymptotically exact in the limit of small $a$, in agreement with the variational approach described in section 4.4. Higher order corrections can be obtained with this approach of strong localized perturbation [99], but there is to date no analytical evaluation of the numerical coefficients.

\subsection{Other approaches}

Another approach to the problem of cyclization has been proposed by Sokolov [97]. Let us denote $r_{\mathrm{ee}}(t)=\left|\mathbf{X}_{\mathrm{ee}}(t)\right|$, and call $q\left(r_{\mathrm{ee}}, t\right)$ the probability density of the variable $r_{\mathrm{ee}}$ in the absence of reaction. The approximation done in [97] consists in approximating the probability of the event 'observing $r_{\mathrm{ee}}=a$ for the first time at time $t$ ' by the probability of the other event 'observing $r_{\mathrm{ee}}=a$ at $t$ given the initial conditions of the problem' [97, 100]. With this approximation, the renewal equation becomes:

$$
q\left(r_{\mathrm{ee}}, t \mid \text { ini }\right) \simeq \int_{0}^{t} \mathrm{~d} t^{\prime} F\left(t^{\prime}\right) q\left(r_{\mathrm{ee}}, t \mid a, t^{\prime} ; \text { ini }\right)
$$

where $F(t)$ is the density of the FPT to the target. If the initial condition is the equilibrium distribution for the chain, one recovers exactly the Wilemski Fixman approximation. For different initial conditions however, equation (130) goes beyond the Wilemski Fixman approximation. Note that the convolution structure of the equations is lost, and the equations have to be solved numerically. It would be interesting to compare this approach with numerical simulations to see to what extent it can capture non-Markovian effects.

Likthman and Marques [100] have also proposed that the exact solution for the FPT density can be approached as the result of successive iterations, the $n^{\text {th }}$ iteration involving integrals of the joint probability distribution of observing $\mathbf{X}_{\mathrm{ee}}$ at $n$ different times. The method is relatively formal, but seems to predict correctly the short time behavior of the FPT distribution.

Yet another approach is the application of perturbative dynamic renormalization group theory [112 116]. The results in this case are derived in the limit of long chains, $N \rightarrow \infty$, and are derived as perturbation in the small parameter $\epsilon=4-d$, where $d$ is the spatial dimension. In the case of the Rouse chain, the results of this theory for the long time decay rate of the density of first-passage time is:

$$
k_{R G}^{-1}=\frac{\pi \zeta N^{2}}{16 k(4-d)} .
$$

It is interesting to compare this approach with the results of the Wilemski Fixman theory. A simple generalization of (98) to the case of a $d$-dimensional space, with $a / L \rightarrow 0$, leads to:

$$
\langle\mathbf{T}\rangle_{W F}=\int_{0}^{\infty} \mathrm{d} t\left\{\frac{L^{d}}{[\psi(t)]^{d / 2}}-1\right\} .
$$

Since the MSD function behaves as $\psi(t) \simeq t^{1 / 2}$ for short times, this integral converges only for $d<4$. The divergence for $d \rightarrow 4$ of (132) is determined by the short time behavior of $\psi$, and reads: 


$$
\langle\mathbf{T}\rangle_{W F} \simeq \frac{\pi N^{2} \zeta}{16(4-d) k}=k_{R G}^{-1} \quad(d \rightarrow 4, N \rightarrow \infty) .
$$

The expression (133) is exactly the expression obtained in the renormalization group theory for a quantity that is slightly different from the reaction time (the inverse of the reaction rate at long times) [117]. The fact that this result of the renormalization group can be derived by developing the reaction time obtained from the Markovian theory suggests that there is a local equilibrium assumption that is made in the renormalization group theory. The advantage of renormalization group theory is that one can treat with the same method the case of polymers with hydrodynamic interactions or excluded volume. Interestingly, for large $N$, the theory predicts that the reaction rate in the case where both excluded volume and hydrodynamic interactions are included scales as the equilibrium contact probability between the reactants, as in a law of mass action.

\section{Concluding remarks and perspectives}

To conclude, let us summarize the main content of this review. We have presented a general theoretical framework which enables the accurate determination of the mean FPT (MFPT) for regular random walks in bounded domains. We then briefly mentioned extensions of these results to related first-passage observables such as splitting probabilities and occupation times. It was showed that this analytical approach provides a universal scaling dependence of the MFPT on both the volume of the confining domain and the source target distance in the case of general Markovian scale-invariant processes. This analysis is applicable to a broad range of stochastic processes characterized by length scale-invariant properties, and reveals the key role that can be played by the starting position of the random walker.

We next showed that such an analysis can be extended to non-Markovian processes, on the example of the Rouse chain (note that the simpler example of persistent random walks was analyzed in [118]). Taking the Rouse model as a paradigm of polymer dynamics, we developed a theory of polymer reaction kinetics that takes into account the non-Markovian features that characterize the dynamics of polymers. This approach shows that non-Markovian effects can be quantitatively important, and that the reaction kinetics crucially depend on the non-equilibrium statistics of polymer conformations at the instant of the reaction. We show quantitatively that the typical reactive conformation of the polymer is more extended than the equilibrium conformation, which leads to reaction times that are significantly shorter than those predicted by existing Markovian theories. These result apply to both reactions with fixed targets, and cyclization reactions. Together, our results provide a better understanding of the complex kinetics of polymer reactions involved, for example, in the formation of loops of RNA or polypeptide chains.

We believe that the results presented in this review open several promising perspectives. First, the analysis of the MFPT in confinement for non-Markovian processes was performed on the example of the Rouse chain. We anticipate that similar methods could be developed to deal with more general Gaussian non-Markovian processes. This includes the very important case of fractional Brownian motion, which is a Gaussian model for anomalous diffusion presenting strongly non-Markovian features. There is a growing number of experimental observations and theoretical works indicating that this kind of stochastic motion is involved in a number of different fields, such as in the motion of telomeres in the nucleus [119], polymer translocation [122, 123], single file diffusion [124] or the motion of particles in complex fluids $[15$ 17, 120, 121]. Determining the first-passage properties for this kind of random processes could therefore find applications in very different fields and calls for a 
generalization of the methods described in this review to more general non-Markovian processes. In the context of polymer physics, the case of more realistic polymer dynamics models, such as semi-flexible chains [125], models involving dihedral interactions [89], the Zimm model [87] that incorporates hydrodynamics interactions, or models of branched polymers [126] also deserve particular interest.

Second, it should be noted that in this review, we focused only on the first moment of the FPT distribution. In general, the distribution of the FPTs can be expected to be quite broad, since trajectories hitting the target range from very short ones when the target is found immediately, to very long ones when the walker explores the whole volume before reaching the target position. Hence, fluctuations can be important in FPT problems [127, 128], and the analysis of the FPT statistics at all time scales requires the determination of the full FPT distribution. This in addition can give access to further first-passage observables, such as participation ratios discussed in [127, 128]. In the case of Markovian walkers, it was demonstrated that the FPT distribution falls into well defined universality classes after a rescaling by the MPFT $[14,24]$, which is therefore a very important determinant of the distribution. It would therefore be very interesting to determine the full distribution of the FPT in confinement for non-Markovian processes, and check whether similar properties still hold.

\section{Acknowledgments}

Support from European Research Council starting Grant FPTOpt-277998 is acknowledged.

\section{References}

[1] van Kampen N G 1992 Stochastic Processes in Physics and Chemistry (Amsterdam: North Holland)

[2] Redner S 2001 A Guide to First Passage Processes (Cambridge: Cambridge University Press)

[3] Metzler R, Oshanin G and Redner S 2014 First Passage Problems: Recent Advances (Singapore: World Scientific)

[4] Rice S A 1985 Diffusion Limited Reactions (Amsterdam: Elsevier)

[5] Tuckwell H C 1988 Introduction to Theoretical Neurobiology (Cambridge: Cambridge University Press)

[6] Doering C R and Gadoua J C 1992 Phys. Rev. Lett. 692318

[7] Bénichou O, Coppey M, Moreau M, Suet P H and Voituriez R 2005 Optimal search strategies for hidden targets Phys. Rev. Lett. 94198101

[8] Bénichou O, Coppey M, Moreau M, Suet P H and Voituriez R 2005 A stochastic model for intermittent search strategies J. Phys.: Condens. Matter. 17427586

[9] Bénichou O, Loverdo C, Moreau M and Voituriez R 2008 Optimizing intermittent reaction paths Phys. Chem. Chem. Phys. 10705972

[10] Bénichou O, Loverdo C, Moreau M and Voituriez R 2011 Intermittent search strategies Rev. Mod. Phys. 8303

[11] Sheinman M, Bénichou O, Kafri Y and Voituriez R 2012 Classes of fast and specific search mechanisms for proteins on DNA Rep. Prog. Phys. 75026601

[12] Condamin S, Bénichou O and Moreau M 2005 First passage times for random walks in bounded domains Phys. Rev. Lett. 95260601

[13] Condamin S, Bénichou O and Moreau M 2007 Random walks and Brownian motion: a method of computation for first passage times and related quantities in confined geometries Phys. Rev. E 75021111

[14] Bénichou O, Chevalier C, Klafter J, Meyer B and Voituriez R Jun 2010 Geometry controlled kinetics Nat. Chem. 24727

[15] Weiss M 2013 Single particle tracking data reveal anticorrelated fractional Brownian motion in crowded fluids Phys. Rev. E $\mathbf{8 8} 010101$ 
[16] Ernst D, Hellmann M, Köhler J and Weiss M 2012 Fractional Brownian motion in crowded fluids Soft Matter 848869

[17] Turiv T, Lazo I, Brodin A, Lev B I, Reiffenrath V, Nazarenko V G and Lavrentovich O D 2013 Effect of collective molecular reorientations on Brownian motion of colloids in nematic liquid crystal Science 34213514

[18] Panja D February 2010 Generalized langevin equation formulation for anomalous polymer dynamics J. Stat. Mech. (2010) L02001

[19] Weber S C, Spakowitz A J and Theriot J A 2010 Bacterial chromosomal loci move subdiffusively through a viscoelastic cytoplasm Phys. Rev. Lett. 104238102

[20] Burnecki K, Kepten E, Janczura J, Bronshtein I, Garini Y and Weron A 2012 Universal algorithm for identification of fractional Brownian motion. a case of telomere subdiffusion Biophys. J. 103 183947

[21] Hajjoul $\mathrm{H}$ et al 2013 High throughput chromatin motion tracking in living yeast reveals the flexibility of the fiber throughout the genome Genome. Res. 23182938

[22] Bray A J, Majumdar S N and Schehr G 2013 Persistence and first passage properties in nonequilibrium systems Adv. Phys. 62225361

[23] Condamin S, Benichou O and Klafter J 2007 First passage time distributions for subdiffusion in confined geometry Phys. Rev. Lett. 98250602

[24] Meyer B, Chevalier C, Voituriez R and Bénichou O 2011 Universality classes of first passage time distribution in confined media Phys. Rev. E 83051116

[25] Hughes B D 1995 Random Walks and Random Environments (Oxford: Oxford University Press)

[26] Noh J D and Rieger H 2004 Constrained spin dynamics description of random walks on hierarchical scale free networks Phys. Rev. E 6903

[27] Aldous D and Fill J 1999 Reversible Markov Chains and Random Walks on Graphs (www.stat. berkeley.edu/users/aldous/RWG/book.html)

[28] Barton G 1989 Elements of Green Functions and Propagation: Potentials, Diffusion and Waves (New York: Oxford University Press)

[29] Haynes C P and Roberts A P 2008 Global first passage times of fractal lattices Phys. Rev. E 78 0411119

[30] Tejedor V, Bénichou O and Voituriez R 2009 Global mean first passage times of random walks on complex networks Phys. Rev. E 80065104

[31] Meyer B, Agliari E, Bénichou O and Voituriez R 2012 Exact calculations of first passage quantities on recursive networks Phys. Rev. E 85026113

[32] Condamin S and Benichou O 2006 Exact expressions of mean first passage times and splitting probabilities for random walks in bounded rectangular domains J. Chem. Phys. 124206103

[33] Van Zon J S, Morelli M J, Tanase Nicola S and Rein ten Wolde P 2006 Diffusion of transcription factors can drastically enhance the noise in gene expression Biophys. J. 91435067

[34] Meyer B, Bénichou O, Kafri Y and Voituriez R 2012 Geometry induced bursting dynamics in gene expression Biophys. J. 102218691

[35] Condamin S, Benichou O and Moreau M 2005 First passage times for random walks in bounded domains Phys. Rev. Lett. 95260601

[36] Godrèche C and Luck J M 2001 Statistics of the occupation time of renewal processes J. Stat. Phys. 104489

[37] Majumdar S M and Comtet A 2002 Local and occupation time of a particle diffusing in a random medium Phys. Rev. Lett. 89060601

[38] Benichou O, Coppey M, Klafter J, Moreau M and Oshanin G 2003 On the joint residence time of $n$ independent two dimensional Brownian motions J. Phys. A: Math. Gen. 36722531

[39] Blanco S and Fournier R 2003 An invariance property of diffusive random walks EPL (Europhysics Letters) 6116873

[40] Bénichou O, Coppey M, Moreau M, Suet P H and Voituriez R 2005 Averaged residence times of stochastic motions in bounded domains EPL (Europhysics Letters) 704248

[41] Condamin S, Benichou O and Moreau M 2005 First exit times and residence times for discrete random walks on finite lattices Phys. Rev. E 72016127

[42] Blanco S and Fournier R 2006 Short path statistics and the diffusion approximation Phys. Rev. Lett. 97230604

[43] Burov S and Barkai E 2007 Occupation time statistics in the quenched trap model Phys. Rev. Lett. 982506014 
[44] Bel G and Barkai E 2005 Weak ergodicity breaking in the continuous time random walk Phys. Rev. Lett. 942406024

[45] Lomholt M A, Zaid I M and Metzler R 2007 Subdiffusion and weak ergodicity breaking in the presence of a reactive boundary Phys. Rev. Lett. 98200603

[46] Metzler R, Jeon J H, Cherstvy A G and Barkai E 2014 Anomalous diffusion models and their properties: non stationarity, non ergodicity, and ageing at the centenary of single particle tracking Phys. Chem. Chem. Phys. 162412864

[47] Ferraro M and Zaninetti L 2004 Physica A 338307

[48] Bouchaud J P and Georges A 1990 Anomalous diffusion in disordered media: statistical mechanisms, models and physical applications Phys. Rep. 195127293

[49] Benichou O, Coppey M, Moreau M and Oshanin G 2005 Kinetics of diffusion limited catalytically activated reactions: an extension of the Wilemski Fixman approach J. Chem. Phys. 123194506

[50] Condamin S, Tejedor V and Benichou O 2007 Occupation times of random walks in confined geometries: from random trap model to diffusion limited reactions Phys. Rev. E 76050102

[51] Ward M J and Keller J B 1993 Strong localized perturbations of eigenvalue problems SIAM J. Appl. Math. 5377098

[52] Cheviakov A F and Ward M J 2011 Optimizing the principal eigenvalue of the Laplacian in a sphere with interior traps Math. Comp. Modeling 531394

[53] Schuss Z, Singer A and Holcman D 2007 The narrow escape problem for diffusion in cellular microdomains Proc. Natl Acad. Sci. USA 10416098103

[54] Schuss Z 2012 The narrow escape problem a short review of recent results J. Sci. Comput. pp 117

[55] Chevalier C, Bénichou O, Meyer B and Voituriez R 2011 First passage quantities of Brownian motion in a bounded domain with multiple targets: a unified approach J. Phys. A: Math. Theor. 44025002

[56] Benichou O and Voituriez R 2008 Narrow escape time problem: time needed for a particle to exit a confining domain through a small window Phys. Rev. Lett. 100168105

[57] Tejedor V, Bénichou O and Voituriez R 2011 Close or connected: Distance and connectivity effects on transport in networks Phys. Rev. E 83066102

[58] Barton G 1989 Elements of Green's Functions and Propagation (Oxford: Oxford Science Publications)

[59] Condamin S, Bénichou O, Tejedor V, Voituriez R and Klafter J 2007 First passage times in complex scale invariant media Nature 4507780

[60] Ben Avraham D and Havlin S 2000 Diffusion and Reactions in Fractals and Disordered Systems (Cambridge: Cambridge University Press)

[61] Montroll E W 1969 Random walks on lattices. III. calculation of first passage times with application to exciton trapping on photosynthetic units J. Math. Phys. 1075365

[62] Erik M 2005 Bollt and ben Avraham D 2005 What is special about diffusion on scale free nets? New J. Phys. 726

[63] Condamin S, Tejedor V, Voituriez R, Benichou O and Klafter J 2008 Probing microscopic origins of confined subdiffusion by first passage observables Proc. National Academy of Sciences 1055675

[64] Metzler R and Klafter J 2000 The random walk's guide to anomalous diffusion: a fractionnal dynamics approach Phys. Rep. 339177

[65] Metzler R and Klafter J 2004 The restaurant at the end of the random walk: recent developments in the description of anomalous transport by fractional dynamics J. Phys. A: Math. Gen. 37 R161

[66] Havlin S and ben Avraham D 1987 Diffusion in disordered media Adv. Phys. 36695

[67] Bunde A and Havlin S (ed) 1991 Fractals and Disordered Systems (Berlin: Springer)

[68] Parson R P and Kopelman R 1982 Percolative versus homogenous energy transport kinetics: time resolved donor and acceptor fluoresence of isotopic mixed naphthalene crystals Chem. Phys. Lett. 8752832

[69] Zimmerman S B and Minton A P 1993 Macromolecular crowding: biochemical, biophysical, and physiological consequences Annu. Rev. Biophys. Biomol. Struc. 2206

[70] Zhou H X, Rivas G and Minton A P 2008 Macromolecular crowding and confinement: biochemical, biophysical, and potential physiological consequences Annu. Rev. Biophys. 3737597

[71] Saxton M J 2007 A biological interpretation of transient anomalous subdiffusion. I. qualitative model Biophys. J. 92117891

[72] Saxton M J 2008 A biological interpretation of transient anomalous subdiffusion. II. reaction kinetics Biophys. J. 9476071 
[73] Malchus N and Weiss M 2010 Elucidating anomalous protein diffusion in living cells with fluorescence correlation spectroscopy facts and pitfalls J. Fluor. 201926

[74] Szymanski J and Weiss M 2009 Elucidating the origin of anomalous diffusion in crowded fluids Phys. Rev. Lett. 1030381024

[75] Bénichou O, Chevalier C, Meyer B and Voituriez R 2011 Facilitated diffusion of proteins on chromatin Phys. Rev. Lett. 106038102

[76] Lebedev D, Filatov M, Kuklin A, Islamov A, Stellbrink J, Pantina R, Denisov Yu, Toperverg B and Isaev Ivanov V 2008 Structural hierarchy of chromatin in chicken erythrocyte nuclei based on small angle neutron scattering: fractal nature of the large scale chromatin organization Crystallogr. Rep. $\mathbf{5 3} 1105$

[77] Bancaud A, Huet S, Daigle N, Mozziconacci J, Beaudouin J and Ellenberg J 2009 Molecular crowding affects diffusion and binding of nuclear proteins in heterochromatin and reveals the fractal organization of chromatin EMBO J. 28378598

[78] Lieberman Aiden E et al 2009 Comprehensive mapping of long range interactions reveals folding principles of the human genome Science $\mathbf{3 2 6} 28993$

[79] Haynes C P and Roberts A P 2009 Generalization of the fractal Einstein law relating conduction and diffusion on networks Phys. Rev. Lett. 1030206014

[80] Roberts A P and Haynes C P 2011 Electrostatic approximation of source to target mean first passage times on networks Phys. Rev. E 83

[81] Reuveni S, Granek R and Klafter J 2010 Vibrational shortcut to the mean first passage time problem Phys. Rev. E 81040103

[82] Benichou O, Meyer B, Tejedor V and Voituriez R 2008 Zero constant formula for first passage observables in bounded domains Phys. Rev. Lett. 101130601

[83] Akkermans E, Benichou O, Dunne G V, Teplyaev A and Voituriez R 2012 Spatial log periodic oscillations of first passage observables in fractals Phys. Rev. E 86061125

[84] Van Kampen N G 1992 Stochastic Processes in Physics and Chemistry 3rd edn (Amsterdam: North Holland)

[85] Panja D 2010 Anomalous polymer dynamics is non Markovian: memory effects and the generalized Langevin equation formulation JSHT (2010) P06011

[86] Démery V, Jacquin H and Bénichou O 2014 Generalized Langevin equations for a driven tracer in dense soft colloids: construction and applications arXiv:1401.5515

[87] Doi M and Edwards S F 1988 The Theory of Polymer Dynamics (Oxford: Clarendon)

[88] Bixon M and Zwanzig R 1978 Optimized Rouse Zimm theory for stiff polymers J. Chem. Phys. 681896902

[89] Dolgushev M and Blumen A 2013 Dynamics of discrete semiflexible chains under dihedral constraints: analytic results J. Chem. Phys. 138204902

[90] Krug J, Kallabis H, Majumdar S N, Cornell S J, Bray A J and Sire C 1997 Persistence exponents for fluctuating interfaces Phys. Rev. E 56270212

[91] Guérin T, Bénichou O and Voituriez R 2013 Reactive conformations and non Markovian kinetics of a Rouse polymer searching for a target in confinement Phys. Rev. E 87032601

[92] Guérin T, Bénichou O and Voituriez R 2012 Non Markovian polymer reaction kinetics Nat. Chem. 456873

[93] Doi M 1975 Diffusion controlled reaction of polymers Chem. Phys. 945566

[94] Wilemski G and Fixman M 1974 Diffusion controlled intrachain reactions of polymers. 1. Theory J. Chem. Phys. 6086677

[95] Wilemski G and Fixman M 1974 Diffusion controlled intrachain reactions of polymers. 2. Results for a pair of terminal reactive groups J. Chem. Phys. 6087890

[96] Szabo A, Schulten K and Schulten Z 1980 FPT approach to diffusion controlled reactions J. Chem. Phys. 7243507

[97] Sokolov I M 2003 Cyclization of a polymer: first passage problem for a non Markovian process Phys. Rev. Lett. 90080601

[98] Toan N M, Morrison G, Hyeon C and Thirumalai D 2008 Kinetics of loop formation in polymer chains J. Phys. Chem. B 1126094106

[99] Amitai A, Kupka I and Holcman D 2012 Computation of the mean first encounter time between the ends of a polymer chain Phys. Rev. Lett. 109108302

[100] Likthman A E and Marques C M 2006 First passage problem for the Rouse polymer chain: an exact solution Europhys. Lett. 759717 
[101] Portman J J 2003 Non Gaussian dynamics from a simulation of a short peptide: loop closure rates and effective diffusion coefficients J. Chem. Phys. 118238191

[102] Portman J J and Wolynes P G 1999 Complementary variational approximations for intermittency and reaction dynamics in fluctuating environments J. Phys. Chem. A 1031060210

[103] Yu Sliusarenko O, Yu Gonchar V, Chechkin A V, Sokolov I M and Metzler R 2010 Kramers like escape driven by fractional Gaussian noise Phys. Rev. E 81041119

[104] Pastor R W, Zwanzig R and Szabo A 1996 Diffusion limited first contact of the ends of a polymer: comparison of theory with simulation J. Chem. Phys. 105387882

[105] Chen J Z Y, Tsao H K and Sheng Y J 2005 Diffusion controlled first contact of the ends of a polymer: crossover between two scaling regimes Phys. Rev. E 72031804

[106] Guérin T, Bénichou O and Voituriez R 2013 Reactive conformations and non Markovian cyclization kinetics of a Rouse polymer J. Chem. Phys. 138094908

[107] Sunagawa S and Doi M 1976 Theory of diffusion controlled intrachain reactions of polymers. 2 Polym. J. 823946

[108] Sunagawa S and Doi M 1975 Theory of diffusion controlled intrachain reactions of polymers Polym. J. 760412

[109] Gardiner C 2004 Handbook of Stochastic Methods for Physics, Chemistry and the Natural Sciences (Berlin: Springer)

[110] Doi M 1975 Theory of diffusion controlled reaction between non simple molecules Chem. Phys. 1110713

[111] Ward M J and Keller J B 1993 Strong localized perturbations of eigenvalue problems SIAM J. Appl. Math. $\mathbf{5 3} 77098$

[112] Friedman B and O'Shaughnessy B 1993 Kinetics of intermolecular reactions in dilute polymer solutions and unentangled melts Macromolecules 26572639

[113] Friedman B and O'Shaughnessy B 1993 Intermolecular reactions in dilute polymer solutions nonexistence of diffusion controlled limit Europhys. Lett. 2366772

[114] Friedman B and O'Shaughnessy B 1993 Theory of intramolecular reactions in polymeric liquids Macromolecules 26488898

[115] Friedman B and O'Shaughnessy B 1991 Short time behavior and universal relations in polymer cyclization $J$. de Physique I 147186

[116] Friedman B and O'Shaughnessy B 1989 Theory of polymer cyclization Phys. Rev. A 4059509

[117] Friedman B and O'Shaughnessy B 1988 Universal behavior in reacting polymer systems Phys. Rev. Lett. 606467

[118] Tejedor V, Voituriez R and Bénichou O 2012 Optimizing persistent random searches Phys. Rev. Lett. 10808810302

[119] Bronstein I, Israel Y, Kepten E, Mai S, Shav Tal Y, Barkai E and Garini Y 2009 Transient anomalous diffusion of telomeres in the nucleus of mammalian cells Phys. Rev. Lett. 103018102

[120] Jeon J H, Tejedor V, Burov S, Barkai E, Selhuber Unkel C, Berg Sørensen K, Oddershede L and Metzler R 2011 In vivo anomalous diffusion and weak ergodicity breaking of lipid granules Phys. Rev. Lett. 106048103

[121] Jeon J H, Leijnse N, Oddershede L B and Metzler R 2013 Anomalous diffusion and power law relaxation of the time averaged mean squared displacement in worm like micellar solutions New J. Phys. 15045011

[122] Palyulin V V, Ala Nissila T and Metzler R 2014 Polymer translocation: the first two decades and the recent diversification Soft Matter 10901637

[123] Panja D, Barkema G T and Kolomeisky A B 2013 Through the eye of the needle: recent advances in understanding biopolymer translocation J. Phys.: Condens. Matter. 25413101

[124] Lizana L, Ambjörnsson T, Taloni A, Barkai E and Lomholt M A 2010 Foundation of fractional Langevin equation: harmonization of a many body problem Phys. Rev. E 81051118

[125] Guérin T, Dolgushev M, Bénichou O, Voituriez R and Blumen A 2014 Cyclization kinetics of Gaussian semiflexible polymer chains Phys. Rev. E 90052601

[126] Dolgushev M, Berezovska G and Blumen A 2011 Branched semiflexible polymers: theoretical and simulation aspects Macromol. Theor. Simul. 2062144

[127] Mejía Monasterio C, Oshanin G and Schehr G 2011 First passages for a search by a swarm of independent random searchers JSHT (2011) P06022

[128] Mattos T G, Mejía Monasterio C, Metzler R and Oshanin G 2012 First passages in bounded domains: when is the mean first passage time meaningful? Phys. Rev. E 86031143 